\title{
A GENERAL FUNCTIONAL EQUATION $\left({ }^{1}\right)$
}

\author{
BY \\ J. H. B. KEMPERMAN
}

1. Introduction. In the following, $E_{m}$ denotes the $m$-dimensional Eucliddean space of points $x=\left(x_{1}, \cdots, x_{m}\right)$ with the usual norm $|x|$. For $B_{1} \subset E_{m}$, $B_{2} \subset E_{m}$ and $\lambda$ real, $B_{1}+B_{2}$ or $\lambda B_{1}$ denotes the set of all the points $b_{1}+b_{2}$ or $\lambda b_{1}$, respectively, $\left(b_{1} \in B_{1}, b_{2} \in B_{2}\right)$. Further, the "measure" of a subset of $E_{m}$ is always meant to be its $m$-dimensional Lebesgue measure. Unless otherwise stated, all functions considered are complex-valued. A function on $E_{m}$ is said to be additive when it satisfies the functional equation $f(x+y)=f(x)$ $+f(y)$. A real-valued additive function on $E_{m}$ is linear, as soon as it is bounded above on a set of positive measure, Ostrowski [15], or on a set $B$, such that some $n$-fold sum $B+\cdots+B$ contains an interior point, cf. $\S 3$. Thus, the following generalization, (cf. $\$ 2$ ), of a theorem due to Steinhaus [20] yields a new proof of Ostrowski's result. Let $B_{1}$ and $B_{2}$ be two subsets of $E_{m}$, each of positive measure; then it is possible to delete sets of measure 0 from $B_{1}$ and $B_{2}$, in such a way that $B_{1}+B_{2}$ becomes an open set.

Let $\Omega$ be the class of all functions $f(x)$ on $E_{m}$ equal to a polynomial, (in the components of $x$ ). Let $b_{0}, b_{1}, \cdots, b_{k}$ be $k+1$ different real numbers and let $f_{0}(x), \cdots, f_{k}(x)$ be a set of $k+1$ functions on $E_{m}$. The central part of this paper consists of results of the following type. (i) If $f_{0}$ is bounded on a (measurable) set of positive measure and, moreover,

$$
\sum_{i=0}^{b} f_{i}\left(x+b_{i} y\right) \in \Omega \quad \text { for each } y \in C,
$$

where $C$ is a subset of $E_{m}$ of positive measure, then $f_{0} \in \Omega$. (ii) Let $\theta_{1}, \theta_{2}$ be a fixed pair of rationally independent real numbers, and let $p, q$ denote arbitrary non-negative integers. If $m=1, f_{0}$ is measurable and $C=\left\{c \mid c=p \theta_{1}+q \theta_{2}\right.$; $p+q \leqq k\}$ then (1) implies the existence of a $g \in \Omega$, such that $f(x)=g(x)$ for almost all $x$.

The above result (i) may be generalized by introducing the notion of a "local condition." Whether or not a function $f(x)$ satisfies a given local condition $P$ at a point $x_{0}$, depends only on the behavior of $f(x)$ in an arbitrarily small neighborhood of $x_{0}$. We only consider local conditions $P$, such that: (a) If $f(x)$ satisfies $P$ at $x_{0}$ then $f(x+h)$ satisfies $P$ at $x_{0}-h$, for each $h \in E_{m}$; (b) If $f_{1}$ and $f_{2}$ satisfy $P$ at $x_{0}$ then also $f_{1}-f_{2}$. For $A$ as an open subset of $E_{m}$,

Presented to the Society, April 14, 1956; received by the editors July 2, 1956.

(1) This work was supported in part by a research grant from the National Science Foundation, NSF-G1979. 
$\Omega_{P}(A)$ will denote the class of all the functions on $A$, which satisfy $P$ at each point in $A$. Thus, $f \in \Omega_{P}(A)$ implies $\left(^{*}\right) \Delta_{h} f=f(x+h)-f(x) \in \Omega_{P}\left(A_{h}\right)$, for each $h \in E_{m}$; here, $A_{h}=A \cap(A-h)$ is the domain of $\Delta_{h} f$. A local condition $P$ is said to have the weak difference property if, vice versa, for $f$ as a function on the open set $A,\left({ }^{*}\right)$ implies $f \in \Omega_{P}(A)$, whenever $f$ is bounded on some subset of $A$ of positive measure. Now, from Theorem 6.1, we have the following generalization of (i). Let $A$ and $C$ be subsets of $E_{m}$, where $A$ is open and connected and $C$ is of positive measure. Let $P$ be a given local condition with the weak difference property. Finally, let $b_{0}=0, b_{1}, \cdots, b_{k}$ be $k+1$ different real numbers, and let $f_{i}(x)(i=0, \cdots, k)$ be a function defined on $A+b_{i} C$. Assertion: if (1) holds for $\Omega=\Omega_{P}(A)$ then $f_{0} \in \Omega_{P}(A)$, whenever $f_{0}$ is bounded on some subset of $A$ of positive measure.

The results of de Bruijn $[2 ; 3]$ imply the weak difference property of the local conditions in $E_{1}$, that $f(x)$ be Riemann integrable, (or continuous, or absolutely continuous, or of bounded variation, or differentiable, respectively), throughout an interval about $x_{0}$. In $\$ 5$, we establish the weak difference property for certain local conditions in $E_{m}$, for instance, the local conditions that $f(x)=f\left(x_{1}, \cdots, x_{m}\right)$ be continuous, (or analytic, or equal to a polynomial, or bounded, respectively), throughout an entire neighborhood of the point $x_{0}$.

In $\S 7$, we concern ourselves with results of the type of the above assertion (ii), some of which are generalizations of results due to Boas [1]. Finally, in $\S 8$, we give a new proof of the following theorem due to Skitovic [19]. Let $a_{j}$ and $b_{j},(j=1, \cdots, n)$, be real constants and let $X_{1}, \cdots, X_{n}$ be independent random variables, such that $\sum a_{j} X_{j}$ and $\sum b_{j} X_{j}$ are independent. Then each $X_{j}$, for which $a_{j} b_{j} \neq 0$, is normally distributed. We note that each of the $\S \S 2,3,7$ and 8 is a self-contained unit with an interest of its own.

Before proceeding, we want to give some counterexamples, showing that the assumptions on $f_{0}$, in the above assertions (i) and (ii), cannot be weakened very much without strengthening the assumptions on $C$, and vice versa $\left({ }^{2}\right)$. The following construction is similar to one given by Erdös and Golomb [6, 16].

Let $U \subset E_{m}$ be a Hamel basis of the linear space $E_{m}$ over the enumerable field $R$, obtained by adjoining $b_{0}, \cdots, b_{k}$ to the field of rational numbers, i.e. each $x \in E_{m}$ has a unique representation as a finite sum $x=\sum H_{\alpha}(x) u_{\alpha}$ with $H_{\alpha}(x) \in R$ and $u_{\alpha} \in U$. Let $u_{0} \in U$ be fixed; then $H(x)=H_{0}(x)$ is additive on $E_{m}$, such that $H\left(b_{i} x\right)=b_{i} H(x), i=0, \cdots, k$. Further, the additive group $G=\{x \mid H(x)=0\}$ is not of positive measure; for, otherwise, $G=G+G$ would contain an interior point, thus, $G=E_{m}$, contradicting $u_{0} \notin G$. But $E_{m}$ is the union of the denumerably many cosets $\{x \mid H(x)=r\}$ of $G,(r \in R)$, hence,

(2) These counterexamples also show, that the same is true for the above stated consequence of Theorem 6.1, provided that each function in $\Omega_{P}(A)$ is measurable on $A$. 
$G$ is a nonmeasurable set of the power $c$. Therefore, $H$ is nonmeasurable, thus, unbounded on each set of positive measure.

Suppose that $k+1 \geqq 3$, and let $\gamma_{0}, \cdots, \gamma_{k}$ be real constants with $\sum \gamma_{i}$ $=\sum b_{i} \gamma_{i}=0$ and $\gamma_{0} \neq 0$. Then the set of functions $f_{i}(x)=\gamma_{i} H(x),(i=0, \cdots, k)$, satisfies

$$
\sum_{i=0}^{k} f_{i}\left(x+b_{i} y\right)=0,
$$

identically in $x$ and $y$. But, $H$ being nonmeasurable, $f_{0}=\gamma_{0} H \notin \Omega$, thus, in assertion (i), the condition on $f_{0}$ cannot be omitted, even if $C=E_{m}$. Further, the set of bounded functions $f_{i}(x)=\gamma_{i}$ arc $\tan H(x),(i=0, \cdots, k)$, satisfies (2) for $x \in E_{m}, y \in G$, though $f_{0} \notin \Omega$. From the properties of $G$, it follows that, in assertion (ii), "measurable" cannot be replaced by "bounded," while, in assertion (i), the condition "of positive measure" on $C$ cannot be replaced by (say) "of positive outer measure."

2. Sums of sets of positive measure. Let $B$ be a measurable subset of the Euclidean space $E_{m}$. The set $B$ is said to be of unit density at the point $x_{0} \in E_{m}$ when

$$
\lim _{r \rightarrow 0} \mu\left(B \cap S_{r}\right) \mu\left(S_{r}\right)^{-1}=1 .
$$

Here, $S_{r}$ denotes the sphere $\left|x-x_{0}\right|<r$, while $\mu(U)$ denotes the $m$-dimensional Lebesgue measure of $U$. By $B^{*}$ we shall denote the set of all the points $x_{0}$ in $B$ at which $B$ is of unit density. Then $B$ is of unit density at almost all points of $B$, (cf. Sierpinsky [17]) thus, $\mu\left(B^{*}\right)=\mu(B)$.

Theorem 2.1. For $i=1, \cdots, k$, let $B_{i}$ be a subset of $E_{m}$ of positive measure and let $a_{i}$ be any fixed point in $B_{i}^{*}$. Then there exist positive numbers $\delta$ and $\eta$, such that

$$
\mu\left(\left(B_{1}-x_{1}\right) \cap \cdots \cap\left(B_{k}-x_{k}\right)\right)>\eta>0,
$$

whenever $\left|x_{i}-a_{i}\right|<\delta, i=1, \cdots, k$.

Proof. Replacing $B_{i}$ by $B_{i}-a_{i}$, we may assume that $a_{i}=0$, thus, $B_{i}$ is of unit density at $0,(i=1, \cdots, k)$. Let $\epsilon$ be a fixed real number, $0<\epsilon<1 / k$, and let $S$ be a fixed sphere $|x|<\rho$ such that

$$
\mu\left(C_{i}\right) \geqq(1-\epsilon) \mu(S) \quad(i=1, \cdots, k),
$$

where

$$
C_{i}=B_{i} \cap S
$$$$
(i=1, \cdots, k)
$$

From $C_{i} \subset B_{i}$

$$
m_{0}=\mu\left(\left(B_{1}-x_{1}\right) \cap \cdots \cap\left(B_{k}-x_{k}\right)\right) \geqq \mu\left(S \cap\left(C_{1}-x_{1}\right) \cap \cdots \cap\left(C_{k}-x_{k}\right)\right) \text {, }
$$


hence, (letting $\bar{D}$ denote the complement of $D$ ).

$$
m_{0} \geqq \mu(S)-\sum_{1}^{k} \mu\left(S \cap\left(\overline{C_{i}-x_{i}}\right)\right) .
$$

Further, from

$$
\begin{aligned}
\mu\left(S \cup\left(C_{i}-x_{i}\right)\right) & =\mu(S)+\mu\left(\bar{S} \cap\left(\overline{C_{i}-x_{i}}\right)\right) \\
& =\mu\left(C_{i}-x_{i}\right)+\mu\left(S \cap\left(C_{i}-x_{i}\right)\right),
\end{aligned}
$$

together with (1) and $C_{i} \subset S$, we have

$$
\begin{aligned}
\mu\left(S \cap \overline{\left(C_{i}-x_{i}\right.}\right) & =\mu(S)-\mu\left(C_{i}\right)+\mu\left(\bar{S} \cap\left(C_{i}-x_{i}\right)\right) \\
& \leqq \epsilon \mu(S)+\mu\left(\bar{S} \cap\left(S-x_{i}\right)\right)=\epsilon \mu(S)+O\left(\left|x_{i}\right|\right) .
\end{aligned}
$$

In fact, one easily proves that, in $E_{m}$,

$$
\mu(\bar{S} \cap(S-x)) \leqq m \rho^{-1} \mu(S)|x| .
$$

From (3), it follows that

$$
m_{0} \geqq\left(1-k_{\epsilon}\right) \mu(S)-O\left(\left|x_{1}\right|\right)-\cdots-O \mid\left(x_{k} \mid\right),
$$

showing that to each positive number $\eta<(1-k \epsilon) \mu(S)$ there corresponds a positive number $\delta$ such that $m_{0}>\eta$ whenever $\left|x_{i}\right|<\delta, i=1, \cdots, k$.

The following result is due to Steinhaus $[20$, p. 99].

Let $B_{1}$ and $B_{2}$ be two linear sets, each of positive measure. Then $B_{1}-B_{2}$ contains an interval of positive length. Moreover, if $B_{1}=B_{2}$ then 0 is an interior point of $B_{1}-B_{2}$.

Theorem 2.1 yields the following generalization.

TheOREM 2.2. Let $B_{1}$ and $B_{2}$ be subsets of $E_{m}$, each of positive measure. Then, after deleting suitable subsets of measure 0 from $B_{1}$ and $B_{2}$, the set $B_{1}-B_{2}$ becomes an open set. In fact, $B_{1}^{*}-B_{2}^{*}$ (and, also, $\left.B_{1}^{*}+B_{2}^{*}\right)$ is open.

More precisely, to each point d in $B_{1}^{*}-B_{2}^{*}$ there correspond positive numbers $\delta$ and $\eta$, such that

$$
|y-d|<\delta \text { implies } \mu\left(B_{1}^{*} \cap\left(B_{2}^{*}+y\right)\right)>\eta \text {. }
$$

Proof. Note that $B_{i}^{*} \subset B_{i}$ and $\mu\left(B_{i}^{*}\right)=\mu\left(B_{i}\right),(i=1,2)$. Let $d=a_{1}-a_{2}$ with $a_{i} \in B_{i}^{*},(i=1,2)$. From Theorem 2.1 , there exist positive numbers $\delta$ and $\eta$, such that

$$
\mu\left(B_{1}^{*} \cap\left(B_{2}^{*}+y\right)\right)=\mu\left(\left(B_{1}-a_{1}\right) \cap\left(B_{2}-a_{1}+y\right)\right)>\eta_{1}
$$

whenever $\left|\left(a_{1}-y\right)-a_{2}\right|=|y-d|<\delta$.

The following lemma will be needed in the proofs of Theorem 4.1 and Theorem 7.1.

LEMMA 2.1. Let $B$ be a subset of $E_{m}$ of positive measure. Let $\left\{\xi_{n}\right\}$ be a se- 
quence of points in $E_{m}$, converging to 0. Let $B^{\prime}$ be the set of all those points $x$ in $B$ for which $x+\xi_{n} \in B$, infinitely often. Then $B^{\prime}$ is of positive measure.

Proof. We may assume that $B$ is a bounded set. We have $0 \in B^{*}-B^{*}$, hence, from Theorem 2.2, there exist positive numbers $\delta$ and $\eta$, such that $\mu(B \cap(B-\xi))>\eta$ whenever $|\xi|<\delta$. Let $C_{n}=B \cap\left(B-\xi_{n}\right)$; then $\mu\left(C_{n}\right)>\eta$ whenever $n>N_{0}$. Further,

$$
B^{\prime}=\bigcap_{N=1}^{\infty} \bigcup_{n=N}^{\infty} C_{n}=\bigcap_{N=1}^{\infty} D_{N}
$$

Here, $\mu\left(D_{N}\right) \geqq \mu\left(C_{N}\right)>\eta$, for $N>N_{0}$, while each set of the decreasing sequence $\left\{D_{N}\right\}$ is a subset of the bounded set $B$. It follows that $\mu\left(B^{\prime}\right) \geqq \eta>0$.

Finally, we mention the following generalization of a result due to Ruziewicz [16].

Let $B$ be a subset of $E_{m}$ of positive measure. Then to each positive integer $k$ there corresponds a positive number $\delta_{k}$, such that, for each $u$ in $E_{m}$ with $|u|<\delta_{k}$, the set $B$ contains an arithmetical progression $b_{1}, b_{2}, \cdots, b_{k}$ with $b_{i}-b_{i-1}=u$, $(i=2, \cdots, k)$.

Proof. We may assume that 0 is a point of unit density of $B$. From Theorem 2.1, applied for $a_{i}=0, B_{i}=B,(i=1, \cdots, k)$, there exists a positive number $\delta$, such that $\mu\left(\left(B-x_{1}\right) \cap \cdots \cap\left(B-x_{k}\right)\right)>0$, whenever $\left|x_{i}\right|<\delta$, $(i=1, \cdots, k)$. Letting $x_{i}=(i-1) u,(i=1, \cdots, k)$ with $(k-1)|u|<\delta$, it follows that the set of points $b_{1}$ with $b_{1}+(i-1) u \in B, i=1, \cdots, k$, is of positive measure, hence, nonempty.

Added in proof. Let $X$ be a locally compact additive group, let $\mu$ denote a regular right Haar measure on $X$, and let $B_{1}, \cdots, B_{k}$ be Borel sets in $X$. It can be shown that there exists a sequence $\left\{U_{n}\right\}$ of neighborhoods of 0 with the following property. Let $\left\{S_{n}\right\}$ be a sequence of Borel sets with $\mu\left(S_{n}\right)>0, S_{n}<U_{n}$; then there exists a Borel set $D$ with $\mu(\bar{D})=0$, such that, for each $x \in D$,

$$
\begin{aligned}
\lim _{n \rightarrow \infty} \mu\left(\left(B_{i}-x\right) \cap S_{n}\right) \mu\left(S_{n}\right)^{-1} & =1 & & \text { if } x \in B_{i}, \\
& =0 & & \text { if } x \in \bar{B}_{i},
\end{aligned}
$$

$i=1, \cdots, k$. Letting $B_{i}^{*}=B_{i} \cap D$, the results and proofs of this section carry over to $X$. Somewhat simpler proofs are obtained by observing that the function $\mu\left(\left(B_{1}-x_{1}\right) \cap \cdots \cap\left(B_{k}-x_{k}\right)\right)$ is jointly continuous in the $x_{i}$, cf. P. R. Halmos, Measure theory, New York, 1950, p. 266.

\section{Additive functions.}

Definition. A subset $B$ of $E_{m}$ is said to be of positive type when a positive integer $n$ can be found, such that the $n$-fold sum $B^{(n)}=B+\cdots+B$ contains a nonempty open subset. If $n$ is the smallest such integer, we put $\nu(B)=1 / n$; when $B$ is not of positive type we put $\nu(B)=0$. 
TheOREM 3.1. Let $H(x)$ be a real-valued additive function on $E_{m}$ and suppose that $H(x)$ is bounded above on a set $B$ of positive type. Then $H(x)$ is of the form $H(x)=a x$, where $a$ is a constant vector in $E_{m}$.

Proof. Let $H(x) \leqq M$ for $x \in B$, where $M$ is a constant. Let $\nu(B)=1 / n$ and let $x \in B^{(n)}$; then $x$ can be written as $x=x_{1}+\cdots+x_{n}$ with $x_{i} \in B$, thus, $H(x)=H\left(x_{1}\right)+\cdots+H\left(x_{n}\right) \leqq n M$. Let $S$ be some open sphere $\left|x-x_{0}\right|<\epsilon$, completely contained in $B^{(n)}$. Then $|x|<\epsilon$ implies $x_{0}+x \in B^{(n)}$ and $x_{0}-x$ $\in B^{(n)}$, hence,

$$
H(x)=H\left(x_{0}+x\right)-H\left(x_{0}\right) \leqq n M-H\left(x_{0}\right)
$$

and $-H(x)=H(-x) \leqq n M-H\left(x_{0}\right)$. Consequently, $|H(x)| \leqq K=n M-H\left(x_{0}\right)$ when $|x|<\epsilon$. The rest is classical. For $|x|<\epsilon / q$, where $q$ is a positive integer, we have

$$
|H(y+x)-H(y)|=|H(x)|=1 / q|H(q x)| \leqq K / q,
$$

thus, $H$ is continuous everywhere. Finally, $H(\lambda x)=\lambda H(x)$ for $\lambda$ rational, hence, for $\lambda$ real.

REMARK. A slight modification of the above proof yields the following more general result. Let $X$ be a topological linear space over the field of the reals, and let $H(x)$ be a real additive function on $X$, which is bounded above on a set of positive type. Then $H(\lambda x)=\lambda H(x)$ for $\lambda$ real. Thus, if an additive function $H$ on $X$ is bounded on a set $B$, with $B-B$ of positive type, then both the real and imaginary part of $H$ is bounded on $B^{\prime}=B \cup(-B)$, which is of positive type, i.e. $H(\lambda x)=\lambda H(x)$ for $\lambda$ real. This implies a result of Kestelman [8] for normed linear spaces.

From Theorem 2.2, if $B$ is a subset of $E_{m}$ of positive measure then $B^{*}+B^{*}$ is open, (thus, $\nu(B) \geqq 1 / 2$ ). Consequently, if a real-valued additive function $H$ on $E_{m}$ is bounded above on a set of positive measure, (e.g. when $H$ is measurable), then $H(x)$ is a linear function. In fact, this result $\left(^{3}\right)$ in $E_{m}$ can be easily obtained from the special case $m=1$, which is due to Ostrowski [15]. However, Theorem 3.1 applies to much thinner sets. For, the following sets $B$ are all of measure 0 but of positive type.

1. In $E_{1}$, the closed set $B$ of all real numbers of the form $\sum_{1}^{\infty} \epsilon_{n} p^{-n}$, where $\epsilon_{n}=0,1$ and where $p$ is a fixed integer $\geqq 3$. Clearly, $\nu(B)=1 /(p-1)$. In general, let $B_{1}$ be any closed set to which there corresponds a denumerable set $C$ in $E_{m}$ such that $E_{m}$ is the union of all the sets $c+B_{1}^{(n)},(c \in C ; n=1,2, \cdots)$. Then $B_{1}$ is of positive type. For, at least one of the closed sets $c+B_{1}^{(n)}$ is not nowhere dense, hence, contains an interior point.

2. In $E_{2}$, the set $B$ of points $(\cos \theta, \sin \theta)$, where $\theta$ runs through a set of real numbers of positive linear measure.

3. In $E_{m}$, the set $B=B_{1} \cup \cdots \cup B_{m}$, where $B_{j}$ is a set of positive linear

(3) It can also easily be deduced from Lemma 2.1, cf. the proof of Theorem 4.1. 
type situated on a straight line $L_{j},(j=1, \cdots, m)$, provided that no hyperplane is parallel to each $L_{j}$; cf. Kestelman $[8,146]$.

4. Difference properties. In this paper, a local condition in $E_{m}$ is defined by a class $P^{*}$ of functions on $E_{m}$ such that $g_{1} \in P^{*}, g_{2} \in P^{*}$, together imply $g_{1}-g_{2} \in P^{*}$. Let $x_{0} \in E_{m}$ and let $f(x)$ be a function defined on an open set containing $x_{0}$. We say that $f(x)$ satisfies at $x_{0}$ the local condition $P$ associated to $P^{*}$ when a function $g \in P^{*}$ and a positive number $\epsilon$ can be found, such that $f(x)=g\left(x-x_{0}\right)$ for $\left|x-x_{0}\right|<\epsilon$, (thus, a restriction imposed on the behavior of $f(x)$ in the immediate neighborhood of $x_{0}$ ). It follows that any local condition $P$ has the following properties: (i) If $f(x)$ satisfies $P$ at $x_{0}$ then, for each $h \in E_{m}$, the function $f(x+h)$ satisfies $P$ at $x_{0}-h$; (ii) If $f_{1}$ and $f_{2}$ satisfy $P$ at $x_{0}$ then also $f_{1}-f_{2}$ and $f_{1}+f_{2}$.

Let $P$ be a local condition and let $A$ be an open subset of $E_{m}$. Then the class of all functions $f$ on $A$, satisfying $P$ at each point of $A$, will be denoted as $\Omega_{P}(A)$. Further, for $h \in E_{m}$, we put

$$
A \cap(A-h)=A_{h} .
$$

Thus, when $f(x)$ is defined on $A$ then $\Delta_{h} f(x)=f(x+h)-f(x)$ is defined on $A_{h}$.

Definition. A local condition $P$ is said to be bounded, (or measurable, respectively), when a function, which satisfies $P$ at a point $x_{0}$, is always bounded, (or measurable, respectively), in some (open) neighborhood of $x_{0}$.

Definition. A local condition is said to have the difference property when the following is true for each open subset $A$ of $E_{m}$. Let $f$ be a function on $A$ such that $\Delta_{h} f \in \Omega_{P}\left(A_{h}\right)$ for each $h \in E_{m}$. Then there exists an additive function $H$ on $E_{m}$, such that $f-I I \in \Omega_{P}(A)$.

Definition. A local condition $P$ is said to have the weak difference property when the following is true for each open subset $A$ of $E_{m}$. Let $f(x)$ be a function on $A$, which is bounded on a subset $B$ of $A$ of positive measure, and such that $\Delta_{h} f \in \Omega_{P}\left(A_{h}\right)$ for each $h \in E_{m}$. Then $f \in \Omega_{P}(A)$.

Definition. A class $\Omega$ of functions on $E_{m}$ is said to have the difference property when to each function $f$ on $E_{m}$, such that $\Delta_{h} f \in \Omega$ for each $h \in E_{m}$, there corresponds an additive function $H$ on $E_{m}$, such that $f-H \in \Omega$.

De Bruijn $[2 ; 3]$ proved the difference property for a great number of classes of functions on $E_{1}$. We mention:

(B1) The class of all polynomials.

(B2) The class of all trigonometric polynomials of period 1 .

(B3) The class of all functions, analytic for $-\infty<x<+\infty$.

(B4) The class of all functions, which can be extended to a function $f(x+i y)$, analytic in the strip $|y| \leqq 1$.

(B5) The class of all real functions with $|f(x)-f(0)| \leqq 1$.

Further, his results easily imply that each of the following "adjectives" represents a (bounded and measurable) local condition in $E_{1}$ having the difference property. (Observe that, for $P$ as a local condition in $E_{m}$ with the 
difference property, the class $\Omega_{P}\left(E_{m}\right)$ always has the difference property.)

(B6) Continuous. (B7) Riemann integrable, (=bounded and continuous almost everywhere). (B8) Absolutely continuous. (B9) Of bounded variation. (B10) $k$ times differentiable. (B11) $k$ times differentiable, $k$ th derivative bounded. (B12) $k$ times differentiable, $k$ th derivative continuous. Here, $k$ is a fixed integer $\geqq 1$.

A function $f(x)$ satisfies such a local condition at the point $x_{0}$ when it is defined and continuous (or Riemann integrable, etc.) throughout an open interval containing $x_{0}$. We note that the local conditions "measurable" and "bounded" do not have the difference property, cf. [2, p. 195, p. 217]. However, the latter condition does have the weak difference property, as is shown by the following theorem.

ThEOREM 4.1. In $E_{m}$, let $P$ be the local condition of boundedness, i.e. a function $f$ satisfies $P$ at a point $x_{0}$ if and only if $f$ is defined and bounded in some neighborhood of $x_{0}$. Then $P$ has the weak difference property.

Proof. Let the function $f$ be defined on an open set $A$, such that $|f(x)| \leqq M$ $=$ constant throughout a subset $B$ of $A$ of positive measure, while $\Delta_{h} f \in \Omega_{P}\left(A_{h}\right)$ for $h \in E_{m}$. Suppose that $f(x)$ does not satisfy $P$ at some point $x_{0}$ in $A$. Then there exists a sequence $\left\{\xi_{n}\right\}$ of points in $E_{m}$, converging to 0 , and such that $\left|f\left(x_{0}+\xi_{n}\right)\right| \rightarrow \infty$ for $n \rightarrow \infty$. From Lemma 2.1 , there exists a point $y_{0}$, $y_{0} \in B^{\prime} \subset B \subset A$, such that $y_{0}+\xi_{n} \in B$ for infinitely many $n$, hence, $\left|f\left(y_{0}+\xi_{n}^{\prime}\right)\right|$ $\leqq M$ for some infinite subsequence $\left\{\xi_{n}^{\prime}\right\}$ of $\left\{\xi_{n}\right\}$. But then, for $h=y_{0}-x_{0}$, we have $\left|\Delta_{h} f\left(x_{0}+\xi_{n}^{\prime}\right)\right| \rightarrow \infty$ for $n \rightarrow \infty$, contradicting the assumption that $\Delta_{h} f(x)$ satisfies $P$ at $x_{0}$.

Lemma 4.1. Let $P$ be a local condition such that: (i) $P$ has the difference property; (ii) $P$ is either bounded or measurable; (iii) If a is a constant vector then the linear function $g(x)=a x$ satisfies $P$ at each point $x_{0}$. Then $P$ has the weak difference property.

Proof. Let the function $f$ be defined as in the preceding proof. $P$ having the difference property, it follows that $f=g+H$ with $g \in \Omega_{P}(A)$ and $H$ additive. It suffices to prove that $H$ is linear; for then, $H \in \Omega_{P}(A)$, hence, $f \in \Omega_{P}(A)$. When $P$ is measurable, $g \in \Omega_{P}(A)$ is measurable on $B$, thus, bounded on a subset $B_{1}$ of $B$ of positive measure. But then the additive function $H$ is bounded on $B_{1}$, implving that $H$ is linear, (cf. $\S 3$ ). When $P$ is bounded, $g(x)$ is bounded on each compact subset of $A$. Hence, letting $B_{1}$ be any compact subset of $B$ of positive measure the same conclusion holds.

REMARK. Condition (iii) is in some sense a necessary condition. For let $P$ be any local condition with the weak difference property and let $g(x)$ be a polynomial (in the components $x_{1}, \cdots, x_{m}$ of $x$ ). Then $g(x)$ satisfies $P$ at each point $x_{0}$, as is easily shown by induction with respect to the degree of $g$. 
5. Local conditions having the difference property. In this section, we shall establish the difference property for certain local conditions $P$ in $E_{m}$. The reader will observe that each of these local conditions $P$ satisfies the assumptions of Lemma 4.1 , thus, $P$ has also the weak difference property. The methods of proof are similar to those of de Bruijn [2]; as to his results, we shall only use the fact that, in $E_{1}$, each of the local conditions of continuity and differentiability has the difference property.

THEOREM 5.1. In $E_{m}$, the (bounded and measurable) local condition of continuity has the difference property.

Proof. Let $A$ be an open subset of $E_{m}$ and let $f(x)=f\left(x_{1}, \cdots, x_{m}\right)$ be defined on $A$, such that $\Delta_{h} f(x)$ is continuous throughout $A_{h}$, for each $h \in E_{m}$. Let $y$ be a fixed point in $A$. Because Theorem 5.1 holds for $m=1$, we have for each integer $j(1 \leqq j \leqq m)$ that there exists an additive function $H_{j}\left(x_{j}\right)$ on $E_{1}$, such that, at the point $y$, the function $f\left(x_{1}, \cdots, x_{m}\right)-H_{j}\left(x_{j}\right)$ is continuous in $x_{j}$. Let $H(x)$ be the additive function on $E_{m}$, defined by

$$
H(x)=H\left(x_{1}, \cdots, x_{m}\right)=H_{1}\left(x_{1}\right)+\cdots+H_{m}\left(x_{m}\right) .
$$

Then, at $y$, the function $g(x)=f(x)-H(x)$ is continuous in each $x_{j}$. From

$$
g(x+h)=g(x)+\Delta_{h} g(x)=g(x)+\Delta_{h} f(x)-H(h),
$$

applied for $h=x^{\prime}-y$, it follows that $g(x)$ is continuous in each $x_{j}$ at every point $x^{\prime}$ in $A$. Now, by a theorem of Hahn [7], there exists a point in $A$ at which $g(x)$ is jointly continuous in $x_{1}, \cdots, x_{m}$. Thus, applying (2) again, $g(x)$ is continuous at each point in $A$.

Theorem 5.2. Let $k$ be a fixed positive integer or $k=\infty$, and let $D$ stand for any differential operator in $E_{m}$ of the form

$$
D=\left(\frac{\partial}{\partial x_{i_{1}}}\right) \cdots\left(\frac{\partial}{\partial x_{i_{p}}}\right) \quad \text { with } 0 \leqq p \leqq k,
$$

$(p<\infty)$. To each such operator $D$ we associate a local condition $P_{D}$ with the weak difference property; (note that the condition $P_{D}$ may be void). Let $g(x)$ be a function defined in a neighborhood of the point $x_{0}$ in $E_{m}$. We say that $g(x)$ satisfies at $x_{0}$ the local condition $Q$ when for each $D$ : (i) $D g$ exists throughout some neighborhood of $x_{0}$; (ii) $D g$ satisfies at $x_{0}$ the local condition $P_{D}$. Assertion: the local condition $Q$ is measurable and has the difference property.

REMARK 1 . We shall say that a function $g(x)$ satisfies at $x_{0}$ the local condition $Q_{1}(k)$ when $D g$ exists throughout a neighborhood of $x_{0}$, for each $D$ of the form (3). Taking each $P_{D}$ as the empty condition, Theorem 5.2 implies that $Q_{1}(k)$ has the difference property. Clearly, $Q_{1}(k)$ is the straightforward generalization to $E_{m}$ of the local condition (B10) in $E_{1}$. Theorem 5.2 also im- 
plies the difference property of similar generalizations to $E_{m}$ of (B11), (using Theorem 4.1), and of (B12), (using Theorem 5.1 and Lemma 4.1).

REMARK 2. Let $\phi(x)=\phi\left(x_{1}, \cdots, x_{m}\right)$ be defined on an open set $A$, such that all its first partial derivatives exist in $A$. Then, throughout $A, \phi(x)$ is continuous in each $x_{j}$, implying (cf. Lebesgue $[10,201]$ ) that $\phi(x)$ is of the $(m-1)$ th Baire class, consequently, $\phi(x)$ is measurable in $A$. This implies a.o. that the above local condition $Q$ is measurable.

Proof of Theorem 5.2. We first show that $Q_{1}(k)$ has the difference property. Let $f(x)=f\left(x_{1}, \cdots, x_{m}\right)$ be defined on an open set $A$, such that, for each $h \in E_{m}$ and each $D$ of the form (3), $D \Delta_{h} f$ exists throughout $A_{h}$. Let $y$ be a fixed point in $A$. Because, in $E_{1}$, the local condition of differentiability has the difference property, there exists an additive function $H_{j}$ on $E_{1}$, such that, at the point $y, f\left(x_{1}, \cdots, x_{m}\right)-H_{j}\left(x_{j}\right)$ is differentiable with respect to $x_{j}$. Let $H(x)$ be the additive function on $E_{m}$, defined by (1), and let $g(x)=f(x)-H(x)$. Then $g(x)$ is differentiable with respect to each $x_{j}$, first at the point $y$, but hence, (applying (2) for $h=x^{\prime}-y$ ), at each point $x^{\prime}$ in $A$. This already shows that $Q_{1}(1)$ has the difference property. Further, from Remark 2, $g(x)$ is a measurable function on $A$.

Let $1 \leqq k_{0}<k \leqq \infty$; it suffices to prove that $D^{\prime} g$ exists in $A$ for each $D^{\prime}$ of order $k_{0}+1$, given that $D g$ exists in $A$ for each $D$ of order $k_{0}$. Thus, let $D$ be a fixed operator of order $k_{0}$; then $D g$ exists as a measurable function on $A$. Moreover,

$$
\Delta_{h} D g=D \Delta_{h} g=D\left(\Delta_{h} f-H(h)\right),
$$

thus, for $i=1, \cdots, m, \partial \Delta_{h} D g / \partial x_{i}$ exists throughout $A_{h}$, for each $h \in E_{m}$. Because $Q_{1}(1)$ has the difference property, we have $D g=g_{1}+H_{1}$, where $H_{1}$ is additive on $E_{m}$ and $g_{1}$ has all its first derivatives throughout $A$. From Remark 2, $g_{1}$ is measurable on $A$; hence, $H_{1}$ is measurable and, thus, linear. Consequently, for each $D$ of order $k_{0}$, all the first derivatives of $D g$ exist in $A$, i.e. $D^{\prime} g$ exist in $A$, for each $D^{\prime}$ of order $k_{0}+1$.

Now, let $f$ be defined on $A$, such that, for each $h \in E_{m}, \Delta_{h} f$ satisfies $Q$ and, thus, $Q_{1}(k)$ throughout $A_{h}$. From the above result, $f=g+H$, where $H$ is additive, while $g$ is a measurable function on $A$ satisfying $Q_{1}(k)$ at each point in $A$. Let $D$ be fixed. Then $D g$ is measurable on $A$, hence, bounded on a subset of $A$ of positive measure. Further, from (4) and the definition of $Q$, we have, for each $h \in E_{m}$, that $\Delta_{h} D g$ satisfies the local condition $P_{D}$ at each point in $A_{h}$. Because $P_{D}$ has the weak difference property, it follows that, for each $D$, the function $D g$ satisfies $P_{D}$ in $A$, i.e., $g$ satisfies $Q$ in $A$.

As follows, we introduce a new local condition $\Lambda$ in $E_{m}$, which generalizes the local conditions (B1), (B3) and (B4) in $E_{1}$. Let $R$ be the set of all the vectors $r=\left(r_{1}, \cdots, r_{m}\right)$ in $E_{m}$ with non-negative integral components $r_{j}$. We call $s=\left(s_{1}, \cdots, s_{m}\right)$ a predecessor of $r \in R$, when for some index $j, 1 \leqq j \leqq m$, we have $s_{i}=r_{i}$ for $i \neq j$ and $s_{j}=r_{j}-1$. Let $\Lambda^{*}$ be a finite or denumerable class of 
"sequences" $\left\{\lambda_{r}\right\}$, with index set $R$, and such that $0 \leqq \lambda_{r} \leqq \infty, \lambda_{0}>0$. We assume that: (i) For each pair of sequences $\left\{\lambda_{r}\right\}$ and $\left\{\lambda_{r}^{\prime}\right\}$ in $\Lambda^{*}$, one can find a sequence $\left\{\lambda_{r}^{\prime \prime}\right\}$ in $\Lambda^{*}$ and a positive constant $c$ with $\lambda_{r}+\lambda_{r}^{\prime} \leqq c \lambda_{r}^{\prime \prime}$, for each $r \in R$; (ii) For each sequence $\left\{\lambda_{r}\right\}$ in $\Lambda^{*}$, one can find a sequence $\left\{\lambda_{r}^{\prime}\right\}$ in $\Lambda^{*}$ and a positive constant $c$, such that to each $r \in R$ with $|r| \geqq 1$ there corresponds a predecessor $s \in R$ of $r$ with $\lambda_{s} \leqq c \lambda_{r}^{\prime}$.

A function $g(x)$, defined in a neighborhood of the point $x_{0} \in E_{m}$, will be said to satisfy the local condition $\Lambda$ at $x_{0}$, when there exist a neighborhood $N$ of $x_{0}$, a sequence $\left\{\lambda_{r}\right\}$ in $\Lambda^{*}$, and a positive constant $c$, such that: (i) All mixed derivatives of $g$ exist and are continuous throughout $N$, (and, thus, are independent of the order in which the single differentiations are performed); (ii) $\left|D_{r} g(x)\right| \leqq c \lambda_{r}$, for $r \in R$ and $x \in N$, where

$$
D_{r}=\left(\frac{\partial}{\partial x_{1}}\right)^{r_{1}} \cdots\left(\frac{\partial}{\partial x_{m}}\right)^{r_{m}}
$$

TheOREm 5.3. The local condition $\Lambda$ has the difference property. Obviously, $\Lambda$ is bounded and measurable.

Proof. Let $A$ be an open subset of $E_{m}$ and let $f(x)$ be defined on $A$, such that, for each $h \in E_{m}, \Delta_{h} f$ satisfies $\Lambda$ at each point of $A_{h}$; without loss of generality, we may assume that $f(x)$ is real-valued. Especially, for $r \in R$ and $h \in E_{m}, D_{r} \Delta_{h} f$ exists and is continuous throughout $A_{h}$. In view of Theorem 5.1 and Lemma 4.1 , Theorem 5.2, applied for $k=\infty$, yields the decomposition $f=g+H$, where $H$ is additive on $E_{m}$, while $D_{r} g$ exists and is continuous throughout $A$, for each $r \in R$.

Let $x_{0}$ be a fixed point in $A$; it suffices to prove that $g(x)$ satisfies $\Lambda$ at $x_{0}$. Let $\epsilon_{1}$ and $\epsilon_{2}$ be positive numbers, such that the sphere $\left|x-x_{0}\right| \leqq \epsilon_{1}+\epsilon_{2}$ is entirely contained in $A$. Let $K$ be the sphere $\left|x-x_{0}\right| \leqq \epsilon_{1}$ and let $S$ be the sphere $|x| \leqq \epsilon_{2}$; then $K \subset A_{h}$ for $h \in S$.

For the moment, let $h$ be fixed, $h \in S$. From the assumptions on $\Delta_{h} f(x)$ $=\Delta_{h} g(x)+H(h)$, we have for each point $\xi \in K \subset A_{h}$ that there exist an open neighborhood $N_{\xi} \subset A_{h}$ of $\xi$, a sequence $\left\{\lambda_{r}(\xi)\right\}$ in $\Lambda^{*}$ and a positive constant $c(\xi)$, such that $\left|D_{r} \Delta_{h} g(x)\right| \leqq c(\xi) \lambda_{r}(\xi)$ for $r \in R, x \in N_{\xi}$. From the compactness of $K$ and property (i) of $\Lambda^{*}$, it follows that to each $h \in S$ there correspond a sequence $\left\{\lambda_{r}\right\}$ in $\Lambda^{*}$ and a positive integer $M$, such that

$$
\left|D_{r} \Delta_{h} g(x)\right| \leqq M \lambda_{r} \quad \text { for } r \in R, x \in K .
$$

For a given sequence $\left\{\lambda_{r}\right\}$ in $\Lambda^{*}$ and a given positive integer $M$, let $S_{j}$ be the set of all the points $h \in S$ for which (5) holds. Then $S$ is the union of all possible sets $S_{j}$. Moreover, $\Lambda^{*}$ being enumerable, there are only denumerably many such sets $S_{j}$. Hence, it is not true that each $S_{j}$ is nowhere dense in $S$, i.e. there exists an $S_{j}$ which is dense in some sphere $S^{\prime}$, contained in $S$, (say) the sphere $\left|x-x_{1}\right| \leqq \delta$ with $\delta>0$. If $\left\{\lambda_{r}\right\}$ and $M$, (which are fixed from now 
on), correspond to this $S_{j}$ then (5) holds for a set of values $h$ dense in $S^{\prime}$. But $D_{r} g(x)$ is continuous in $A$; thus, for $x \in K, h \in S^{\prime}$, (hence, $x \in A, x+h \in A$ ), $D, \Delta_{h} g(x)$ is a continuous function of $h$. It follows that (5) holds for each $h \in S^{\prime}$.

Let $x, y$ be any pair of points in $K$ with $|x-y| \leqq 2 \delta$. Applying (5) for $h=h_{1}=x_{1}+2^{-1}(y-x) \in S^{\prime}$ and also for $h=h_{2}=x_{1}-2^{-1}(y-x) \in S^{\prime}$, we have from $x+h_{1}=y+h_{2}$ that

$$
\left|D_{r} g(y)-D_{r} g(x)\right|=\left|D_{r} \Delta_{h_{1}} g(x)-D_{r} \Delta_{h_{2}} g(y)\right| \leqq 2 M \lambda_{r},
$$

for each $r \in R$. Let $M^{\prime}$ be the smallest integer $\geqq \epsilon_{1} / \delta ; K$ being the sphere $\left|x-x_{0}\right| \leqq \epsilon_{1}$, we conclude that

$$
\left|D_{r} g(y)-D_{r} g(x)\right| \leqq 2 M M^{\prime} \lambda_{r} \quad \text { if } r \in R, x \in K, y \in K .
$$

Let $r \in R,|r| \geqq 1$, and let $s$ be any predecessor of $r$, thus, $D_{r} g=\partial D_{s} g / \partial x_{j}$ for some $j, 1 \leqq j \leqq m$. Let $e_{j}$ be the unit vector parallel to the $x_{j}$-axis. Applying (6) for $r$ replaced by $s$ and $x=x_{0}-\epsilon_{1} e_{j}, y=x_{0}+\epsilon_{1} e_{j}$, it follows from the mean value theorem that (for each predecessor $s$ of $r$ ) there exists a point $y \in K$, such that

$$
\left|D_{r} g(y)\right| \leqq M M^{\prime} \lambda_{s} / \epsilon_{1} \text {. }
$$

Combining (6) and (7), we obtain that, for each $r \in R$ with $|r| \geqq 1$, and any predecessor $s$ of $r$,

$$
\left|D_{r} g(x)\right| \leqq M M^{\prime}\left(2 \lambda_{r}+\lambda_{s} / \epsilon_{1}\right) \quad \text { if } x \in K .
$$

Further, for $r=0$, the continuous function $D_{r} g(x)=g(x)$ is certainly bounded on $K$. We now conclude from the properties of the class $\Lambda^{*}$ that there exist a sequence $\left\{\lambda_{r}^{\prime}\right\}$ in $\Lambda^{*}$ and a positive constant $c$ such that $\left|D_{r} g(x)\right| \leqq c \lambda_{r}^{\prime}$ for $r \in R, x \in K$. This shows that $g(x)$ satisfies at $x_{0}$ the local condition $\Lambda$.

We shall now exhibit some applications of Theorem 5.3. As is easily verified, each of the following classes $\Lambda^{*}$ satisfies the required properties (i) and (ii). From Theorem 5.3, for each such $\Lambda^{*}$, the corresponding local condition $\Lambda$ has the difference property, i.e. when $A$ is an open subset of $E_{m}$ and the function $f$ is defined on $A$, such that $\Delta_{h} f \in \Omega_{\Lambda}\left(A_{h}\right)$ for $h \in E_{m}$, then $f-H \in \Omega_{\Lambda}(A)$ for some additive function $H$ on $E_{m}$. Consequently, the class $\Omega_{\Lambda}\left(E_{m}\right)$, of all the functions on $E_{m}$ satisfying $\Lambda$ everywhere, has the difference property.

1. Let $\Lambda^{*}$ consist of the sequences $\left\{\lambda_{r}^{(n)}\right\}=\left\{\lambda_{r_{1}, \ldots, r_{m}}^{(n)}\right\},(n=0,1, \ldots)$, with $\lambda_{r}^{(n)}=1$ if $r_{1}+\cdots+r_{m} \leqq n, \lambda_{r}^{(n)}=0$, otherwise. Then $g \in \Omega_{\Lambda}(A)$ if and only if $g(x)$ is equal to a polynomial throughout each (connected) component of $A$. Thus, we have a.o. that the class $\Omega_{\Lambda}\left(E_{m}\right)$ of all polynomials on $E_{m}$ has the difference property; this generalizes the corresponding result (B1) in $E_{1}$, due to de Bruijn [2].

2. Let $\Lambda^{*}$ consist of the sequences $\left\{\lambda_{r}^{(N)}\right\},(N=1,2, \cdots)$, with 


$$
\lambda_{r}^{(N)}=\lambda_{r_{1}}^{(N)} \cdots, r_{m}=r_{1} ! \cdots r_{m} ! N^{r_{1}+\cdots+r_{m}} .
$$

Then $g \in \Omega_{\Lambda}(A)$ if and only if, throughout each (connected) component of $A$, $g(x)=g\left(x_{1}, \cdots, x_{m}\right)$ is an analytic function of the real variables $x_{1}, \cdots, x_{m}$. Thus we have a.o. that the class $\Omega_{\Lambda}\left(E_{m}\right)$, of all the analytic functions on $E_{m}$, has the difference property; this generalizes the corresponding result (B3) due to de Bruijn [2].

3 . Let $R_{1}, \cdots, R_{m}$ be fixed positive numbers, and let $\Lambda^{*}$ consist of all the sequences $\left\{\lambda_{r}^{(N)}\right\},(N=1,2, \cdots)$, of the form

$$
\lambda_{r_{1}}^{(N)} \cdots, r_{m}=r_{1} ! \cdots r_{m} !\left(R_{1}+1 / N\right)^{-r_{1}} \cdots\left(R_{m}+1 / N\right)^{-r_{m}} .
$$

Then $g \in \Omega_{\Lambda}(A)$ if and only if the following is true for each convex subset $A^{\prime}$ of $A$. In the space of $m$ complex variables $z_{j}=x_{j}+i y_{j},\left(x_{j}, y_{j}\right.$ real $\left.; j=1, \cdots, m\right)$, let $A^{\prime \prime}$ be the set of points satisfying

$$
\left|z_{j}-x_{j}^{\prime}\right| \leqq R_{j}, \quad j=1, \cdots, m \text {, for some }\left(x_{1}^{\prime}, \cdots, x_{m}^{\prime}\right) \in A^{\prime} .
$$

Then there exists a single-valued function $\bar{g}(x+i y)=\bar{g}\left(z_{1}, \cdots, z_{m}\right)$ on $A^{\prime \prime}$, analytic in the complex variables $z_{1}, \cdots, z_{m}$, such that $\bar{g}(x)=g(x)$ for $x \in A^{\prime}$. It follows that the class $\Omega_{\Lambda}\left(E_{m}\right)$, of all analytic functions on $E_{m}$, extendable to an analytic function of $z_{1}, \cdots, z_{m}$ in the region $\left|y_{j}\right| \leqq R_{j}(j=1, \cdots, m)$, has the difference property; this generalizes the corresponding result (B4) in $E_{1}$, due to de Bruijn [2]. As an easy consequence, the class of "entire" functions on $E_{m}$ has the difference property.

4. Let $m=1$, and let $\Lambda^{*}$ consist of the sequences $\left\{N^{r} B_{r}\right\},(N=1,2,3, \cdots)$, where $\left\{B_{r}\right\}$ is a a fixed sequence of positive numbers with $B_{r}>\epsilon^{r} B_{r-1}, \epsilon$ being a positive constant. Then $g \in \Omega_{\Lambda}(A)$ if and only if $\left|D_{r} g(x)\right|^{1 / r}=O\left(B_{r}^{1 / r}\right)$, uniformly in any finite closed subinterval of $A$. Note that the class $\Omega_{\Lambda}(A)$ is quasi-analytic if and only if $A$ is an open interval and

$$
\sum_{r=0}^{\infty}\left[\sup _{s \geq r} B_{s}^{-1 / s}\right]=\infty,
$$

cf. Carleman [4]. The latter condition holds, for instance, when $B_{r} \leqq r$ ! for infinitely many positive integers $r$.

6. A functional equation. Let $\phi(x, y)$ be a function on $E_{m} \times E_{m}$, admitting the following representation as a sum of functions of one variable:

$$
\phi(x, y)=\sum_{i=0}^{k} f_{i}\left(a_{i} x+b_{i} y\right),
$$

$a_{i}, b_{i}$ being given real constants with

$$
a_{i} \neq 0, \quad a_{i} b_{j}-a_{j} b_{i} \neq 0, \quad(i, j=0, \cdots, k ; i \neq j) .
$$

For $k+1 \geqq 3$, this representation is certainly not unique. For, let $\gamma_{0}, \cdots, \gamma_{k}$ 
be real constants, not all zero, with $\sum a_{i} \gamma_{i}=\sum b_{i} \gamma_{i}=0$, and let $H$ be an additive function on $E_{m}$ with $H\left(a_{i} x\right)=a_{i} H(x), H\left(b_{i} x\right)=b_{i} H(x),(i=0, \cdots, k)\left({ }^{4}\right)$. Then $\sum \gamma_{i} H\left(a_{i} x+b_{i} y\right)=0$, hence, if $f_{i}(x)(i=0, \cdots, k)$ is a solution of (1) then also $f_{i}(x)+\gamma_{i} H(x)(i=0, \cdots, k)$. But $H(x)$ is, in general, unbounded on each set of positive measure, hence, the same is true for most solutions of (1).

In order to rule out such pathological solutions, we might consider only those solutions $f_{i}(x)(i=0, \cdots, k)$ of $(1)$, for which each function $f_{i}(x)$ is bounded on some set of positive measure. Assertion (i) of the following Theorem 6.1, (applied for $A=C=E_{m}$ ), easily implies that, for each such solution of (1), all the functions $f_{i}(x)$ are necessarily continuous, (or analytic, or polynomial, etc.), whenever, for each $y \in E_{m}$, the function $\phi_{y}(x)=\phi(x, y)$ is continuous, (or analytic, or polynomial, respectively). Clearly, this is a generalization of the following classical result, (cf. §3). Let $f(x)$ be a function on $E_{m}$, such that: (i) $f(x)$ is bounded on a set of positive measure; (ii) $f(x)$ is a solution of the functional equation

$$
f(y)=f(x+y)-f(x) .
$$

Then $f(x)$ is continuous on $E_{m}$, in fact, $f(x)$ is a (linear) polynomial.

Theorem 6.1. In $E_{m}$, let $A$ be an open connected set and let $C$ be a set of positive measure. Let $\phi(x, y)$ be a given function on $A \times C$ and consider the relation

$$
\phi(x, y)=\sum_{i=0}^{k} f_{i}\left(x+b_{i} y\right)
$$

where $k \geqq 0$ is a given integer and the $b_{i}$ are given real numbers, $b_{i} \neq b_{0}=0$ $(i=1, \cdots, k)$. By a solution of (2) we mean a set of functions $f_{i}(x)$ $(i=0,1, \cdots, k), f_{i}(x)$ being defined on $A+b_{i} C$, such that (2) holds for all pairs of points $x, y$ with $x \in A, y \in C$. Let $f_{i}(x)(i=0, \cdots, k)$ and $f_{i}^{*}(x)(i=0, \cdots, k)$ be two solutions of (2), such that each of the two functions $f_{0}(x)$ and $f_{0}^{*}(x)$ is bounded on some subset of $A$ of positive measure.

Assertion: (i) Let $P$ be a local condition in $E_{m}$ having the weak difference property. Suppose that, for each $y_{0} \in C$, the function $\phi\left(x, y_{0}\right)$ satisfies $P$ at each point of $A$. Then $f_{0}(x)$ satisfies $P$ at each point of $A$.

(ii) If, in addition to the assumptions of (i), $P$ is either bounded or measurable, then $f_{0}(x)-f_{0}^{*}(x)$ is a polynomial on $A$ of degree $\leqq k-1$.

(iii) Suppose that, for each $y_{0} \in C$, the function $\phi\left(x, y_{0}\right)$ is a polynomial on $A$ of degree $\leqq n,\left(n\right.$ fixed). Then $f_{0}(x)$ is a polynomial on $A$ of degree $\leqq n+k$.

Here, the condition, that $C$ be of positive measure, cannot be weakened very much, cf. $\S 1$. In assertion (iii) we allow the value $n=-1$, provided that a

( $)$ One way of obtaining such a function $H$ is by means of a Hamel basis of $E_{m}$ over the field $R$, obtained by adjoining the $a_{i}$ and $b_{i}$ to the field of rational numbers, cf. $\S 1$. 
polynomial of degree -1 is interpreted as the zero-function. Observe that (ii) is a consequence of (i) and (iii). For, suppose that the conditions of (ii) hold. From (i), $f_{0}$ and $f_{0}{ }^{*}$, thus, $f_{0}^{\prime}=f_{0}-f_{0}{ }^{*}$ satisfy $P$ throughout $A$. Because $P$ is bounded or measurable, $f_{0}^{\prime}$ is bounded on a subset of $A$ of positive measure. Further, $f_{i}^{*}=f_{i}-f_{i}^{\prime}(i=0, \cdots, k)$ is a solution of $(2)$ with $\phi(x, y)$ replaced by 0 , a polynomial of degree -1 . Hence, from (iii), $f_{0}^{\prime}$ is a polynomial on $A$ of degree $\leqq k-1$.

We shall say that a function $f(x)$ satisfies $Q$ at a point $x_{0}$, when $f(x)$ is defined and equal to a polynomial throughout an entire neighborhood of $x_{0}$. Clearly, $Q$ is a bounded local condition. From application 1 of Theorem 5.3, $Q$ has the difference property. Hence, from Lemma 4.1, $Q$ has also the weak difference property. Now, suppose that the conditions of assertion (iii) hold. Then (i), (applied to $Q$ instead of $P$ ), already shows that $f_{0}$ satisfies $Q$ throughout $A$, i.e. $f_{0}$ is a polynomial on $A$. Consequently, it suffices to prove (i) and the part of (iii) which asserts that the polynomial $f_{0}$ is of degree $\leqq n+k$.

Proof of Theorem 6.1. From $b_{0}=0$, everything is obvious when $k=0$. Let $k$ be a fixed integer, $k \geqq 1$, and suppose that Theorem 6.1 holds when $k$ is replaced by $k-1$. Let $f_{i}(x)(i=0, \cdots, k)$ be a solution of $(2)$, such that $f_{0}(x)$ is bounded on a subset $B$ of $A$ of positive measure. Let $x_{0}$ be an arbitrary but fixed point in $A$ and let $\xi_{0}$ be any point of unit density of $B, \xi_{0} \in A$. Select any polygonal path (of finite length) $L$ in $A$ with endpoints $\xi_{0}$ and $x_{0}$, and let $\epsilon$ denote the distance between $L$ and the complement of $A, 0<\epsilon \leqq \infty$. Finally, let $A^{\prime}$ be the set of all the points in $E_{m}$ having a distance $<\epsilon / 2$ from $L$. Then: (a) $A^{\prime}$ is open and connected, $x_{0} \in A^{\prime}$; (b) For each $h \in E_{m}$ with $|h| \leqq \epsilon / 2$, we have $A^{\prime}+h \subset A$, especially, $A^{\prime} \subset A$; (c) The function $f_{0}(x)$ is bounded on the subset $B^{\prime}=A^{\prime} \cap B$ of $A^{\prime}$, where $B^{\prime}$ is of positive measure.

From Theorem 2.2, applied for $d=0$, (or from the fact that $B^{\prime}$ and $C$ possess points of unit density), there exists a positive number $\delta, \delta \leqq \epsilon / 2$, such that, for each $h \in E_{m}$ with $|h| \leqq \delta$, both sets $B^{\prime}(h)=B^{\prime} \cap\left(B^{\prime}-h\right)$ and $C(h)$ $=C \cap\left(C+h / b_{k}\right)$ are of positive measure; (note that $b_{k} \neq 0$ ). For the moment, let $h \in E_{m}$ be arbitrary but fixed, $|h| \leqq \delta$. Then, $B^{\prime}(h)$ and $C(h)$ are of positive measure and $A^{\prime}+h \subset A$. Further, let

$$
h_{i}=\left(1-b_{i} / b_{k}\right) h, \quad g_{i}(x)=f_{i}\left(x+h_{i}\right)-f_{i}(x), \quad(i=0, \cdots, k) .
$$

Here, $h_{k}=0$ and, (by $b_{0}=0$ ), $h_{0}=h$, thus, $g_{k}(x)=0$ and $g_{0}(x)=\Delta_{h} f(x)$. Moreover, $x \in B^{\prime}(h)$ implies $x \in B^{\prime}$ and $x+h \in B^{\prime}$, hence, $g_{0}(x)$ is bounded on $B^{\prime}(h)$, where $B^{\prime}(h) \subset B^{\prime} \subset A^{\prime}$. Observe that, for $i=0, \cdots, k$, the function $g_{i}(x)$ is well-defined on $A^{\prime}+b_{i} C(h)$. For, from $A^{\prime} \subset A, A^{\prime}+h \subset A$ and the definition of $C(h)$, it follows that $x \in A^{\prime}+b_{i} C(h)$ implies $x \in A+b_{i} C$ and, also, $x+h_{i} \in A^{\prime}$ $+b_{i}\left(C+h / b_{k}\right)+h_{i}=A^{\prime}+h+b_{i} C \subset A+b_{i} C$.

Let $y^{\prime}$ be any point in $C(h)$. Then $y^{\prime} \in C$ and $y^{\prime}-h / b_{k}=y^{\prime \prime} \in C$, while, from (3), 


$$
b_{i} y^{\prime \prime}+h=b_{i} y^{\prime}+h_{i},
$$$$
(i=0, \cdots, k) .
$$

Under the conditions of assertion (i), (denoted as "case (i)"), both functions $\phi\left(x, y^{\prime}\right)$ and $\phi\left(x, y^{\prime \prime}\right)$ satisfy $P$ throughout $A$, hence, (from $A^{\prime}+h \subset A, A^{\prime} \subset A$ ), the function

$$
\psi\left(x, y^{\prime}\right)=\phi\left(x+h, y^{\prime \prime}\right)-\phi\left(x, y^{\prime}\right)
$$

satisfies $P$ throughout $A^{\prime}$. Similarly, under the conditions of assertion (iii), (denoted as case (iii)), $\psi\left(x, y^{\prime}\right)$ is a polynomial on $A^{\prime}$ of degree $\leqq n$. Finally, from (2), (3), (4), (5) and $g_{k}(x)=0$,

$$
\psi\left(x, y^{\prime}\right)=\sum_{i=0}^{k-1} g_{i}\left(x+b_{i} y^{\prime}\right), \quad\left(x \in A^{\prime}, y^{\prime} \in C(h)\right) .
$$

From $b_{i} \neq b_{0}=0,(i=0, \cdots, k-1)$, and the fact that $g_{0}(x)$ is bounded on the subset $B^{\prime}(h)$ of $A^{\prime}$ of positive measure, it now follows by induction, (replacing $A, C$ and $f_{i}(x), i=0, \cdots, k$, by $A^{\prime}, C(h)$ and $g_{i}(x), i=0, \cdots, k-1$, respectively): (a) In case (i), that $g_{0}(x)=\Delta_{h} f_{0}(x)$ satisfies $P$ throughout $A^{\prime}$; (b) In case (iii), that $\Delta_{h} f(x)$ is a polynomial on $A^{\prime}$ of degree $\leqq n+k-1$. In fact, the latter conclusions hold for any $h \in E_{m}$ with $|h| \leqq \delta$.

Now, suppose first that case (i) holds. Let $h \in E_{m}$ and $x_{1} \in A^{\prime}$ be such that $x_{1}+h=y_{1} \in A^{\prime}$. Because $A^{\prime}$ is an open, connected set, there exists a sequence of points $x_{2}, \cdots, x_{p}, x_{p+1}=y$ in $A^{\prime}$, such that, for $h_{j}=x_{j+1}-x_{j}$, we have $\left|h_{j}\right| \leqq \delta, j=1, \cdots, p$. Thus, the identity

$$
\Delta_{h} f_{0}(x)=\Delta_{h_{1}} f_{0}(x)+\Delta_{h_{2}} f_{0}\left(x+h_{1}\right)+\cdots+\Delta_{h_{p}} f_{0}\left(x+h_{1}+\cdots+h_{p-1}\right)
$$

implies that $\Delta_{h} f_{0}(x)$ satisfies $P$ at $x_{1}$, (for each $h \in E_{m}$ and each $x_{1} \in A_{h}^{\prime}$ ). Because $P$ has the weak difference property, and $f_{0}(x)$ is bounded on the subset $B^{\prime}$ of $A^{\prime}$ of positive measure, it follows that $f_{0}(x)$ satisfies $P$ throughout $A^{\prime}$, especially at the point $x_{0} \in A^{\prime}$. Here, $x_{0}$ was chosen as an arbitrary point in $A$, hence, $f_{0}(x)$ satisfies $P$ throughout $A$. This proves assertion (i).

Now, suppose that case (iii) holds. From assertion (i) and the remark preceding this proof, $f_{0}(x)$ is a polynomial on $A$. Furthermore, for each $h \in E_{m}$ with $|h| \leqq \delta, \Delta_{h} f_{0}(x)$ is a polynomial on $A^{\prime}$ of degree $\leqq n+k-1$. Consequently, $f_{0}(x)$ is a polynomial of degree $\leqq n+k$.

Let $P$ be a given local condition in $E_{m}$. Suppose that the conditions of Theorem 6.1, together with $\phi\left(x, y_{0}\right) \in \Omega_{P}(A)$ for each $y_{0} \in C$, always imply $f_{0} \in \Omega_{P}(A)$. Then $P$ has the weak difference property, showing that the condition of assertion (i) is, in some sense, a necessary condition.

For, let $A$ be any open set in $E_{m}$, and let $f$ be defined on $A$, such that: (i) For each $h \in E_{m}$, and each $x_{0} \in A$ with $x_{0}+h \in A$, the function $\Delta_{h} f$ satisfies $P$ at $x_{0}$; (ii) $f$ is bounded on a subset $B$ of $A$ of positive measure. Let $x_{1}$ be a fixed point of unit density for $B, x_{1} \in A$, having a distance $\epsilon$ from the complement of $A$. Let $A^{\prime}$ be the open sphere $\left|x-x_{1}\right|<\epsilon / 2$ and let $C$ be the set of 
positive measure $|x| \leqq \epsilon / 2$. Then, for each $y \in C$, the function

$$
\phi(x, y)=-\Delta_{y} f(x)=f(x)-f(x+y)
$$

satisfies $P$ throughout $A^{\prime}$. Moreover, $f$ is bounded on the subset $B^{\prime}=A^{\prime} \cap B$ of $A^{\prime}$ of positive measure. From the above assumption concerning $P$, we have $f \in \Omega_{P}\left(A^{\prime}\right)$, especially, $f$ satisfies $P$ at the point $x_{1} \in A^{\prime} \subset A$. Applying $f(x+h)$ $=f(x)+\Delta_{h} f(x)$ for $h=x_{2}-x_{1}$, it follows that $f$ satisfies $P$ at each point $x_{2} \in A^{\prime}$. This shows that $P$ has the weak difference property.

As an interesting consequence of Theorem 6.1 , we have

Theorem 6.2. Let $P$ be a bounded, or measurable, local condition in $E_{m}$ having the weak difference property. Let $b_{0}, b_{1}, \cdots, b_{k}$ be given real numbers with $b_{i} \neq b_{0},(i=1, \cdots, k)$. Let $f_{i}(x)(i=0, \cdots, k)$ be functions on the open connected subset $A$ of $E_{m}$, such that $f_{0}(x)$ is bounded on the subset $B$ of $A$ of positive measure and that, for each $y \in C$, the function

$$
\phi(x, y)=\sum_{i=0}^{k} f_{i}\left(x+b_{i} y\right)
$$

satisfies $P$ at each point $x_{n}$, where it is defined, (i.e. $x_{0}+b_{i} y \in A$ for all $i$ ). Here, $C$ is a subset of $E_{m}$, such that, to each point $x_{0} \in A$, there corresponds a point $c \in E_{m}$ at which $C$ is of unit density and with $x_{0}+\left(b_{i}-b_{0}\right) c \in A$ for $i=1, \cdots, k$; (example: $C$ is of unit density at 0 ). Then the function $f_{0}(x)$ satisfies $P$ throughout $A$.

Proof. Let $A^{*}$ be the set of all the points $x_{0} \in A$, such that, within each neighborhood of $x_{0}$, one can find a set of positive measure on which $f_{0}(x)$ is bounded. Clearly, $A^{*}$ is closed relative to $A$. Further, $A^{*}$ is nonempty, because $x_{0} \in A^{*}$ for each point $x_{0} \in A$ at which $B$ is of unit density.

Let $x_{0}$ be a fixed point in $A^{*}$. Then there exists a point $c_{0}$ in $C$, at which $C$ is of unit density, and such that $x_{i}=x_{0}+\left(b_{i}-b_{0}\right) c_{0} \in A,(i=1, \cdots, k)$. Because $A$ is open, there exist positive numbers $\delta_{1}$ and $\delta_{2}$, such that $\left|x-x_{0}\right|<\delta_{1}$ and $\left|c-c_{0}\right|<\delta_{2}$ together imply $x+\left(b_{i}-b_{0}\right) c \in A,(i=1, \cdots, k)$. Let $A_{0}$ be the open sphere $\left|x-x_{0}\right|<\delta_{1}$ and let $C_{0}$ be the set of all the points $c$ in $C$ with $\left|c-c_{0}\right|<\delta_{2}$. Then $C_{0}$ is of positive measure. Further, from $x_{0} \in A^{*}$, the function $f_{0}(x)$ is bounded on some subset of $A_{0}$ of positive measure. Finally, the function

$$
\psi(x, y)=\phi\left(x-b_{0} y, y\right)=\sum_{i=0}^{k} f_{i}\left(x+\left(b_{i}-b_{0}\right) y\right)
$$

is defined on $A_{0} \times C_{0}$ and, thus, satisfies property $P$ throughout $A_{0}$ for each fixed $y \in C_{0}$. From assertion (i) of Theorem 6.1, $f_{0}(x)$ satisfies $P$ throughout $A_{0}$, especially, at $x_{0} \in A_{0}$. Moreover, $P$ being bounded or measurable, $f_{0}(x)$ is bounded or measurable on each compact subset of $A_{0}$, consequently, each point of $A_{0}$ belongs to $A^{*}$. It follows that the set $A^{*}$ is open, hence, $A^{*}=A$, 
because $A$ is connected. Hence, $f_{0}(x)$ satisfies $P$ at each point $x_{0}$ of $A=A^{*}$.

7. Characteristic sets. In this section, for convenience, we restrict ourselves to the case $m=1$ and to functions $f(x)$ defined for all $x \in E_{1}$. Let $\Omega$ be a class of functions on $E_{1}$, containing all constant functions, and such that $f_{1} \in \Omega, f_{2} \in \Omega$ imply $f_{1}-f_{2} \in \Omega$ and $f_{1}(x+h) \in \Omega$, for each $h \in E_{1}$. Let $(\sim)$ denote a fixed equivalence relation defined on $\Omega$, such that $f_{1} \sim g_{1}, f_{2} \sim g_{2}$ imply $f_{1}-f_{2} \sim g_{1}-g_{2}$ and $f_{1}(x+h) \sim g_{1}(x+h)$, for each $h \in E_{1}$. Further, we suppose that, for a constant function $f, f \sim 0$ implies $f=0$. As an important example, we shall write $f_{1} \approx f_{2}$ if and only if $f_{1}(x)$ and $f_{2}(x)$ differ only on a set of measure 0 .

Definition. A set $S$ of real numbers is said to be characteristic (with respect to $\Omega$ ) when the following two implications hold. (i) Let $f \in \Omega$ be such that $\Delta_{h} f \sim 0$, for each $h \in S$. Then $f$ is equivalent to a constant. (ii) Suppose that to each $h \in S$ there corresponds a function $g_{h} \in \Omega$, in such a way that $\Delta_{h} g_{h^{\prime}}$ $=\Delta_{h^{\prime}} g_{h}$ for $h, h^{\prime} \in S$. Then there exists a $g \in \Omega$ and constants $a_{h}$, such that $\Delta_{h} g \sim g_{h}-a_{h}$, for each $h \in S$.

Any set $S^{\prime}$, containing a characteristic set $S$, is itself a characteristic set. (i) Let $f \in \Omega$ be such that $\Delta_{h} f \sim 0$ for $h^{\prime} \in S^{\prime}$. Then $\Delta_{h} f \sim 0$ for $h \in S$, hence, $f$ is equivalent to a constant. (ii) For $h^{\prime} \in S^{\prime}$, let $g_{h}{ }^{\prime} \in \Omega$ be such that $\Delta_{h} g_{h^{\prime}}$ $=\Delta_{h^{\prime}} g_{h}$ when $h, h^{\prime} \in S^{\prime}$. By $S \subset S^{\prime}$, there exists a $g \in \Omega$ with $\Delta_{h} g-g_{h} \sim-a_{h}$ $=$ constant, when $h \in S$. For $h^{\prime} \in S^{\prime}$ and each $h \in S, \Delta_{h}\left(\Delta_{h^{\prime}} g-g_{h^{\prime}}\right) \sim \Delta_{h^{\prime}}\left(g_{h}-a_{h}\right)$ $-\Delta_{h} g_{h^{\prime}}=0$, hence, $\Delta_{h^{\prime}} g-g_{h^{\prime}}$ is equivalent to a constant $-a_{h^{\prime}}$.

If $C$ is a set of real numbers then the additive group of real numbers generated by $C$ will be denoted as $C \dagger$. Here, $C \dagger$ is dense in $E_{1}$ when, for instance, $C$ contains a pair of rationally independent real numbers.

THEOREM 7.1. Let $C$ be a set of real numbers, such that $C \dagger$ is a characteristic set. Let $f(x)$ be any function on $E_{1}$, such that $\Delta_{h} f \in \Omega$ for each $h \in C$.

Assertion. (i) There exists $a g \in \Omega$ and an additive function $H$ on $E_{1}$, such that $\Delta_{h}(f-g-H) \sim 0$, for each $h \in C \dagger$.

(ii) Suppose that, in $\Omega, f_{1} \sim f_{2}$ implies $f_{1}=f_{2}$, and that to $\Omega$ there corresponds at least one characteristic set. Then the class $\Omega$ has the difference property.

(iii) Suppose that: (a) Each function in $\Omega$ is measurable; (b) In $\Omega, f_{1} \sim f_{2}$ implies $f_{1} \approx f_{2}$. (c) $C \dagger$ is dense in $E_{1}$. Provided that $f(x)$ is bounded on some set of positive measure, we have $f \approx \beta x+g+g^{*}$, where $\beta$ is a constant, $g \in \Omega$, and $g^{*}$ is $a$ bounded function with $\Delta_{h} g^{*} \approx 0$ for $h \in C \dagger$. Provided that $f(x)$ is measurable, we have $f \approx \beta x+g$, where $\beta$ is a constant and $g \in \Omega$.

Proof. It follows from

$$
\Delta_{h-h^{\prime}} f(x)=\Delta_{h} f(x)-\Delta_{h^{\prime}} f\left(x+h-h^{\prime}\right),
$$

that $\Delta_{h} f \in \Omega$, for each $h \in C \dagger$. Let $g_{h}=\Delta_{h} f$, hence, $\Delta_{h} g_{h^{\prime}}=\Delta_{h^{\prime}} g_{h}$, for each $h, h^{\prime}$; moreover, $g_{h} \in \Omega$ when $h \in C \dagger$. Because $C \dagger$ is characteristic, there exists a $g \in \Omega$ and constants $H(h)$, such that $\Delta_{h} g \sim g_{h}-H(h)$ when $h \in C \dagger$. Let $f^{\prime}=f-g$, 
thus, $\Delta_{h} f^{\prime}=\Delta_{h} f-\Delta_{h} g \sim H(h)$ for $h \in C \dagger$. From (1), applied to $f^{\prime}$ instead of $f$, $H(h)$ is an additive function on $C \dagger$; it is easy to extend $H$ to an additive function on the entire group $E_{1}$. Now, for $h \in C \dagger, \Delta_{h}(f-g-H)=\Delta_{h} f^{\prime}-H(h) \sim 0$, proving assertion (i).

Under the assumptions of assertion (ii), $E_{1}$ is characteristic. Hence, applying assertion (i) to $C=C \dagger=E_{1}$, we have that $\Delta_{h} f \in \Omega$, for each $h \in E_{1}$, implies the existence of a $g \in \Omega$ and an additive function $H$, such that $\Delta_{h}(f-g-H)=0$, for each $h \in E_{1}$. Hence, $f-g-H=a=$ constant, thus, $f-H=g+a \in \Omega$.

Suppose that the assumptions of assertion (iii) hold. Let $g, f^{\prime}=f-g$ and $H$ be as in the proof of assertion (i). Here, $f$ is either measurable or $f$ is bounded on some set of positive measure. Further, $g$ is measurable, hence, $f^{\prime}$ is bounded on some bounded set $B^{\prime}$ of positive measure:

$$
\left|f^{\prime}(x)\right| \leqq M
$$

for $x \in B^{\prime}$,

where $M$ is a positive real constant. Moreover, for $h \in C \dagger$., we have $\Delta_{h} f^{\prime} \sim H(h)$, hence, $\Delta_{h} f^{\prime} \approx H(h)$. We want to prove that

$$
h \cdot H\left(h^{\prime}\right)=h^{\prime} \cdot H(h),
$$

for $h, h^{\prime} \in C \dagger$. Because $I$ 's additive, (3) certainly holds when $h$ and $h^{\prime}$ are rationally dependent. On the other hand, let $h$ and $h^{\prime}$ be rationally independent elements of $C \dagger$, such that $h H\left(h^{\prime}\right) \neq h^{\prime} H(h)$. Then there exist two sequences of integers $\left\{\lambda_{j}\right\}$ and $\left\{\mu_{j}\right\}(j=1,2, \cdots)$, such that $h_{j}=\lambda_{j} h+\mu_{j} h^{\prime}$ satisfies $h_{j} \rightarrow 0$ and $\left|H\left(h_{j}\right)\right|=\left|\lambda_{j} H(h)+\mu_{j} H\left(h^{\prime}\right)\right| \rightarrow \infty$ for $j \rightarrow+\infty$. From $h_{j} \in C \dagger$, we have $\Delta_{h_{j}} f^{\prime} \approx H(h)$, i.e.

$$
f^{\prime}\left(x+h_{j}\right)-f^{\prime}(x)=H\left(h_{j}\right),
$$

except for those values $x$ which are in a set $D_{j}$ of measure $0,(j=1,2, \cdots)$. Let $B^{\prime \prime}$ be the set of all those points $x$ in $B^{\prime}$, for which $x \in D_{j}(j=1,2, \cdots)$. Clearly, $B^{\prime \prime}$ is of positive measure. From Lemma 2.1 , there exists a point $x \in B^{\prime \prime}$ such that $x+h_{j} \in B^{\prime \prime}$, infinitely often. Hence, from (2) and (4), $\left|H\left(h_{j}\right)\right| \leqq 2 M$, infinitely often, contradicting $\left|H\left(h_{j}\right)\right| \rightarrow+\infty$. This proves (3) for $h, h^{\prime} \in C \dagger$, thus, $H(h)=\beta h$ for $h \in C \dagger$, where $\beta$ is a constant.

Let $g^{*}=f-\beta x-g=f^{\prime}-\beta x$, thus,

$$
\Delta_{h} g^{*}=\Delta_{h} f^{\prime}-\beta h=\Delta_{h} f^{\prime}-H(h) \approx 0,
$$

for each number $h$ in the everywhere dense set $C \dagger$. Further, $g^{*}$ is bounded on the (bounded) set $B^{\prime}$ of positive measure, while $g^{*}$ is measurable whenever $f$ is measurable. Assertion (iii) now follows from the following result due to Boas [1], applied to $g^{*}$ instead of $f$.

Lemma 7.1. Let $f$ be a function on $E_{1}$, such that $\Delta_{h} f \approx 0$, for each number $h$ in a set dense in $E_{1}$. If $f(x)$ is bounded on a set of positive measure then $f \approx f_{1}$, where $f_{1}$ is some bounded function. Further, if $f(x)$ is measurable then $f \approx \alpha$, where $\alpha$ is a constant. 
This lemma is optimal in some sense. For, under the assumption of the continuum hypothesis, Sierpinsky [18] constructed a bounded nonmeasurable function (with values 0 and 1 ), such that $\Delta_{h} f \approx 0$ for each number $h$. This remark is due to P. Erdös, cf. [2, p. 195].

In applying Theorem 7.1, our main concern is to find sets $S$ which are characteristic with respect to $\Omega$. Because each superset of a characteristic set is characteristic, it is important to find (so-called minimal) characteristic sets, for which no proper subset is characteristic. For instance, a characteristic set consisting of a single element $h$ is certainly minimal; in this case, the number $h$ will be said to be characteristic. Any set, containing a characteristic number, is necessarily a characteristic set.

In the remaining part of this section, we shall apply Theorem 7.1 to the following classes $\Omega_{j}(j=1, \cdots, 4)$ of continuous functions, in each of which equivalence is defined as a strict equality, (and also to certain related classes $\left.\Omega_{j}^{*}\right)$. The proof, that the following stated numbers are indeed characteristic, will be given at the end of this section. Then, from assertion (ii) of Theorem 7.1 , we have that each class $\Omega_{j}(j=1, \cdots, 4)$ has the difference property. For $i=1$, the latter result is due to de Bruijn [2].

(I) Let $\Omega_{1}$ be the class of all polynomials on $E_{1}$. It is easily shown that each number $h \neq 0$ is characteristic. Hence, a set $S$ is characteristic if and only if it contains a nonzero element.

(II) I.et $\Omega_{2}$ be the class of all functions $f$ on $E_{1}$, such that: (i) All derivatives $f^{(n)}$ of $f$ exist; (ii) For $x \rightarrow+\infty$,

$$
f^{(n)}(x)=O\left(x^{\left.-\alpha_{n}\right),} \quad(n=0,1, \cdots),\right.
$$

where $\left\{\alpha_{n}\right\}$ is a nondecreasing sequence of real numbers, depending on $f$, and converging to $+\infty$. Again, each number $h \neq 0$ is a characteristic number.

(III) Let $\rho$ and $\sigma$ be fixed positive numbers. Let $\Omega_{3}$ be the class of all the analytic functions $f(x)$ on $E_{1}$, which can be extended to an entire function $f(z)=f(x+i y)$ with

$$
f(x+i y)=O(\exp (\rho|x|+\sigma|y|)) .
$$

We assert that a number $h$ is characteristic if and only if $0<\sigma|h|<2 \pi$.

(IV) Let $\delta$ be a fixed real number, $\delta \geqq 0$. Let $\Omega_{4}$ be the class of all the analytic functions $f(x)$ on $E_{1}$, periodic of period 1 , which can be extended to an analytic function $f(z)=f(x+i y)$ in the strip $|y| \leqq \delta$.

Let $\Gamma$ denote the set of all the real numbers $h$, to which there corresponds a positive number $\epsilon$, such that the inequality $|n h-m|<e^{-\epsilon n}$ holds for infinitely many pairs of integers $m, n$ with $n \geqq 1$. Then $h \in \bar{\Gamma}$ implies $r_{1} h+r_{2} \in \Gamma$ for each pair of rational numbers $r_{1}, r_{2}$; especially, $\bar{\Gamma}$ contains all rational numbers. Further, $\bar{\Gamma}$ is of measure 0 , in fact, even of Hausdorff dimension 0 . Let $\Gamma$ be the complement of $\bar{\Gamma}$ (with respect to $E_{1}$ ). Then $\Gamma$ contains all irrational 
algebraic numbers and, further, such numbers as $\pi$ and $e$. All these results are well-known, cf. Koksma [9].

We assert that a number $h$ is characteristic with respect to $\Omega_{4}$ if and only if $h \in \Gamma$. Hence, a set $S$ with $S \cap \Gamma \neq 0$ is always a characteristic set.

Lemma 7.2. Suppose that $\Omega$ is a class of continuous functions, on which equivalence is defined as a strict equality. Let $\Omega^{*}$ be the class of all functions $f^{*}$ on $E_{1}$ to which there corresponds an $f \in \Omega$ with $f^{*} \approx f$. Finally, in $\Omega^{*}, f_{1} \sim f_{2}$ if and only if $f_{1} \approx f_{2}$. Assertion: a set of real numbers is characteristic with respect to $\Omega^{*}$ if and only if it is characteristic with respect to $\Omega$.

Proof. If $f_{1} \in \Omega, f_{2} \in \Omega, f_{1} \approx f_{2}$, then $f_{1}=f_{2}$, from the continuity of the function $f_{1}-f_{2}$. This remark enables us to prove Lemma 7.2 in a straightforward manner; we omit the details.

Let $\Omega_{j}^{*}(j=1, \cdots, 4)$ be the class associated to $\Omega_{j}$ in the sense of Lemma 7.2. It follows from Lemma 7.2 that the above assertions, concerning the characteristic numbers of $\Omega_{j}$, apply equally well to the characteristic numbers of $\Omega_{j}^{*},(j=1, \cdots, 4)$. Especially, when $C$ is a pair of rationally independent real numbers then $C \dagger$ is characteristic for $\Omega_{j}{ }^{*}$, provided that, for $j=4$, the set $C \nmid \cap \Gamma$ is nonempty.

Theorem 7.2. Let $j$ be fixed, $(j=1, \cdots, 4)$, and let $\Omega^{*}=\Omega_{j}^{*}$. Let $f_{i}(x)$, $(i=0, \cdots, k)$, be a set of $k+1$ functions on $E_{1},(k \geqq 0)$, such that $f_{0}(x)$ is measurable, and that the function

$$
\phi_{y, y^{\prime}}(x)=\sum_{i=0}^{k} f_{i}\left(x+\lambda_{i} y+\lambda_{i}^{\prime} y^{\prime}\right)
$$

belongs to $\Omega^{*}$, for each pair of non-negative integers $y, y^{\prime}$ with $y+y^{\prime} \leqq k$. Here, $\lambda_{i}, \lambda_{i}^{\prime}(i=0, \cdots, k)$ are real constants, such that, for $i=1, \cdots, k$, the numbers $\lambda_{i}-\lambda_{0}$ and $\lambda_{i}^{\prime}-\lambda_{0}^{\prime}$ are rationally independent, while, for $j=4$, either $\lambda_{i}-\lambda_{0}$ or $\lambda_{i}^{\prime}-\lambda_{0}^{\prime}$ belongs to $\Gamma$.

Assertion: there exists a polynomial $P$, such that $f_{0}-P \in \Omega^{*}$. If $j \neq 4$ we may take $P=0$, hence, $f_{0} \in \Omega^{*}$.

Proof. The assertion is obvious when $k=0$. Let $k$ be a fixed integer, $k \geqq 1$, and suppose that the theorem holds when $k$ is replaced by $k-1$. Let

$$
h=\lambda_{k}-\lambda_{0} \quad \text { and } \quad h^{\prime}=\lambda_{k}^{\prime}-\lambda_{0}^{\prime} .
$$

Then $h, h^{\prime}$ are rationally independent, while, for $j=4$, either $h$ or $h^{\prime}$ is in $\Gamma$. Further, let

$$
g_{i}(x)=f_{i}\left(x+\lambda_{k}\right)-f_{i}\left(x+\lambda_{i}\right), \quad(i=0, \cdots, k) ;
$$

then $g_{0}(x)$ is measurable and $g_{k}(x)=0$. We have, for each pair of non-negative integers $y, y^{\prime}$ with $y+y^{\prime} \leqq k-1$, that the function 


$$
\sum_{i=0}^{k-1} g_{i}\left(x+\lambda_{i} y+\lambda_{i}^{\prime} y^{\prime}\right)=\phi_{y, y^{\prime}}\left(x+\lambda_{k}\right)-\phi_{y+1, y^{\prime}}(x)
$$

belongs to $\Omega^{*}$. Let us first assume that $j \neq 4$. Then, by induction, $g_{0}(x)$ $=\Delta_{h} f_{0}\left(x+\lambda_{0}\right) \in \Omega^{*}$, hence, $\Delta_{h} f_{0} \in \Omega^{*}$. In a similar way, $\Delta_{h} f_{0} \in \Omega^{*}$. Let $C$ be the set, consisting of the two rationally independent numbers $h, h^{\prime}$. Then $C \dagger$ is dense in $E_{1}$ and, moreover, characteristic with respect to $\Omega^{*}=\Omega_{j}{ }^{*}$, $(j \neq 4)$. Because $f_{0}$ is measurable, it follows from assertion (iii) of Theorem 7.1 that $f_{0}=\beta x+g$, where $\beta$ is a constant and $g \in \Omega^{*}$. But $\Omega^{*}$ contains each polynomial, hence, $f_{0} \in \Omega^{*}$.

Now, let $j=4$. From (5), by induction, there exists a polynomial $P_{1}$, such that $g_{0}-P_{1} \in \Omega_{4}^{*}$. By $g_{0}(x)=\Delta_{h} f_{0}\left(x+\lambda_{0}\right)$, we have $\Delta_{h} f_{0}-P=g \in \Omega_{4}^{*}$, for some polynomial $P$. Similarly, $\Delta_{h} f_{0}-P^{\prime}=g^{\prime} \in \Omega_{4}^{*}$, for some polynomial $P^{\prime}$. Thus,

$$
\Delta_{h} \Delta_{h^{\prime}} f_{0}=\Delta_{h^{\prime}}(g+P)=\Delta_{h}\left(g^{\prime}+P^{\prime}\right),
$$

or,

$$
\Delta_{h} g^{\prime}-\Delta_{h^{\prime}} g=\Delta_{h^{\prime}} P-\Delta_{h} P^{\prime}=u \text { (say). }
$$

From $g \in \Omega_{4}^{*}, g^{\prime} \in \Omega_{4}^{*}$, it follows that $u$ is a polynomial of period 1 , such that $\int_{0}^{1} u(x) d x=0$. Consequently, $u(x)=0$. Let $Q$ be any polynomial, such that $\Delta_{h} Q=P$. Then $\Delta_{h}\left(\Delta_{h^{\prime}} Q-P^{\prime}\right)=\Delta_{h^{\prime}} P-\Delta_{h} P^{\prime}=u=0$, thus, $\Delta_{h^{\prime}} Q-P^{\prime}=a=$ constant. Let $f=f_{0}-Q$; then $\Delta_{h} f=\Delta_{h} f_{0}-P=g \in \Omega_{4}^{*}$ and $\Delta_{h^{\prime}} f=\Delta_{h} f_{0}-P^{\prime}-a$ $=g^{\prime}-a \in \Omega_{4}^{*}$. Thus, $f$ is a measurable function with $\Delta_{h} f \in \Omega_{4}^{*}$ and $\Delta_{h} f \in \Omega_{4}^{*}$.

Let $C$ be the set, consisting of the two rationally independent numbers $h$ and $h^{\prime}$, where $h \in \Gamma$ or $h^{\prime} \in \Gamma$. Hence, $C \dagger$ is dense in $E_{1}$ and characteristic with respect to $\Omega_{4}^{*}$. From assertion (iii) of Theorem 7.1, we have $f=\beta x+g^{*}$, where $\beta$ is a constant and $g^{*} \in \Omega_{4}^{*}$. Finally, $f_{0}-(Q+\beta x)=f-\beta x=g^{*} \in \Omega_{4}^{*}$, where $Q+\beta x$ is a polynomial.

Corollary 7.2. Let $j$ be fixed, $(j=1, \cdots, 4)$, and let $\Omega^{*}=\Omega_{j}^{*}$. Let $f_{i}(x)$ $(i=0, \cdots, k)$, be functions on $E_{1}$, such that $f_{0}(x)$ is measurable, $f_{0}(x+1)$ $-f_{0}(x) \approx 0$, while

$$
\phi_{y}(x)=\sum_{i=0}^{k} f_{i}\left(x+\lambda_{i} y\right) \in \Omega^{*},
$$

for $y=0,1, \cdots, k$. Here, $\lambda_{i}(i=0, \cdots, k)$ are real constants such that, for $j \neq 4, \lambda_{i}-\lambda_{0}$ is irrational and, for $j=4, \lambda_{i}-\lambda_{0} \in \Gamma,(i=1, \cdots, k)$. Then $f_{0} \in \Omega^{*}$.

Proof. Let $y^{\prime}$ be an arbitrary integer. Then $f_{0}\left(x+y^{\prime}\right) \approx f_{0}(x)$, hence, for $y=0, \cdots, k$,

$$
\phi_{y}\left(x+y^{\prime}\right)=\sum_{i=0}^{k} f_{i}\left(x+\lambda_{i} y+\lambda_{i}^{\prime} y^{\prime}\right) \in \Omega^{*},
$$

where $\lambda_{0}^{\prime}=0, \lambda_{i}^{\prime}=1,(i=1, \cdots, k)$. From Theorem $7.2, f_{0} \in \Omega^{*}$ when $j \neq 4$. 
Further, for $j=4, f_{0}-P=g \in \Omega^{*}=\Omega_{4}^{*}$, where $P$ is some polynomial. But $P=f_{0}-g$ is equivalent to a function of period 1 , hence, $P$ is constant, thus, $f_{0}=g+P \in \Omega_{4}^{*}$.

REMARK 1 . Given $k+1 \geqq 2$ real numbers $\lambda_{i}(i=0, \cdots, k)$, one can always find a system of $k+1$ bounded, nonmeasurable functions $f_{0}(x), \cdots, f_{k}(x)$, each of period 1 , and such that

$$
\sum_{i=0}^{k} f_{i}\left(x+\lambda_{i} y\right)=0
$$

for each $y$ in some nondenumerable additive group $C \dagger$. This follows from the construction in $\S 1$, replacing $R$ by the field, obtained by adjoining the $\lambda_{i}$ to the field of rational numbers and taking $1 \in U, u_{0} \neq 1$. Thus, in view of $0 \in \Omega_{j}^{*}$ and $f_{0} \in \Omega_{j}^{*}$, the condition of the above corollary, that $f_{0}$ be measurable, cannot be weakened very much, unless we require that (6) holds for each number $y$ in (say) a set of positive measure, cf. Theorem 6.1.

REMARK 2. Let $\Omega^{*}=\Omega_{j}^{*},(1 \leqq j \leqq 4)$, and let $f(x)$ be a measurable function on $E_{1}$, such that $\Delta_{1} f \approx 0$ and

$$
f(u+x)+f(u-x) \in \Omega^{*},
$$

for two values $u_{1}$ and $u_{2}$ of $u$ with an irrational difference $u_{1}-u_{2}$ when $j \neq 4$, and $u_{1}-u_{2} \in \Gamma$ when $j=4$; then $f \in \Omega^{*}$. This result is an immediate consequence of the above corollary, applied for $k=1, f_{0}(x)=f\left(u_{1}+x\right), f_{1}(x)=f(u-x)$, $\lambda_{0}=-\lambda_{1}=u_{2}-u_{1}$. Moreover, if $j=1$ or $j=2$, we have that $f \in \Omega^{*}$ and $\Delta_{1} f \approx 0$ together imply $f \approx a=$ constant. The above for $j=1$ and $j=4$ yields a generalization of some results due to Boas [1, Theorems 1,4].

It remains to verify the assertions (I)-(IV) concerning the characteristic numbers of $\Omega_{j},(j=1, \cdots, 4)$. Note that, for each of these classes, equivalence is defined as a strict equality.

(I) Let $h$ be any real number $\neq 0$. If $f \in \Omega_{1}$ and $\Delta_{h} f=0$ then the polynomial $f$ is constant. Further, for each $g_{1} \in \Omega_{1}$, there exists a polynomial $g$ with $\Delta_{h} g=g_{1}$. Hence, $h$ is characteristic with respect to $\Omega_{1}$.

(II) Let $h$ be any real number $\neq 0$. Let $f \in \Omega_{2}$ be such that $\Delta_{h} f=0$. For $n$ sufficiently large, we have for $x \rightarrow+\infty$ that $f^{(n)}(x)=O\left(x^{-\alpha_{n}}\right)$ with $\alpha_{n}>0$. Hence, from $\Delta_{h} f^{(n)}=0, f^{(n)}=0$, showing that $f$ is a periodic polynomial and, thus, a constant. Further, let $g_{1} \in \Omega_{2}$; then the so-called principal solution $g$ of the difference equation $\Delta_{h} g=g_{1}$ exists and is in $\Omega_{2}$, cf. Nörlund $[14$, p. 56], showing that $h$ is characteristic with respect to $\Omega_{2}$. In fact, let $g_{1}^{(n)}(x)=O\left(x^{-\beta_{n}}\right)$ when $x \rightarrow+\infty,(n=0,1, \cdots)$. Then this principal solution satisfies

$$
g^{(m)}(x)=a_{m}-\sum_{j=0}^{\infty} g_{1}^{(m)}(x+j h),
$$

( $a_{m}=$ constant), for each non-negative integer $m$ with $\beta_{m}>1$, thus, $g^{(m+1)}(x)$ $=O\left(x^{-\beta_{m+1}}\right)$. 
(III) Let $h$ be any real number with $0<\sigma|h|<2 \pi$. Let $f \in \Omega_{3}$ be such that $\Delta_{h} f=0$. Then

$$
g(\exp (2 \pi i z / h))=f(z)
$$

defines a function $g(\zeta)$ which is single valued and analytic for $\zeta \neq 0$. Moreover,

$$
g(\zeta)=O\left(|\zeta|^{\beta}+|\zeta|^{-\beta}\right) \quad \text { with } \beta=\frac{\sigma|h|}{2 \pi}<1 .
$$

Consequently, $g$ and, hence, $f$ is a constant function. Further, let $g_{1} \in \Omega_{3}$. Then

$$
g(x)=\frac{1}{2 \pi i h} \int_{-1 / 2-i \infty}^{-1 / 2+i \infty}\left\{\int_{0}^{x+h w} g_{1}(\xi) d \xi\right\}\left(\frac{\pi}{\sin \pi w}\right)^{2} d w
$$

defines a function $g \in \Omega_{3}$, such that $\Delta_{h} g=g_{1}$, cf. Nörlund [14, p. 78]. This shows that $h$ is characteristic w.r.t. $\Omega_{3}$. On the other hand, let $\sigma|h| \geqq 2 \pi$. Then $f=\exp (2 \pi i x / h)$ satisfies $f \in \Omega_{3}$ and $\Delta_{h} f=0$, showing that the number $h$ is not characteristic.

(IV) Let $h$ be any number in $\Gamma$, thus, $h$ is irrational. Moreover, to each positive number $\epsilon$ there corresponds a positive constant $K(\epsilon)$ with $|n h-m|$ $\geqq K(\epsilon) e^{-\epsilon|n|}$, for each pair of integers $m, n$ with $n \neq 0$. Let $n$ be any integer $\neq 0$ and let $m$ be the integer closest to $n h$. Then $|\sin \pi n h| \geqq 2|n h-m|$, hence,

$$
\left|e^{2 \pi i n h}-1\right|^{-1}=|\sin \pi n h|^{-1} / 2 \leqq(4 K(\epsilon))^{-1} e^{\epsilon|n|},
$$

for each integer $n \neq 0$. Now, let $f \in \Omega_{4}$ be such that $\Delta_{h} f=0$. Because $h$ is irrational and $\Delta_{1} f=0$, we have $\Delta_{h}, f=0$ for an everywhere dense set of values $h^{\prime}=\lambda+\mu h,(\lambda, \mu=$ integers $)$. Further, $f$ is continuous on $E_{1}$, hence, $f$ is a constant function.

Let $g_{1}$ be any function in $\Omega_{4}$, in other words,

$$
g_{1}(x)=\sum_{-\infty}^{\infty} c_{n} e^{2 \pi i n x},
$$

where $\left\{c_{n}\right\}$ is a sequence of complex numbers, satisfying

$$
c_{n}=O\left(e^{-(2 \pi \delta+\eta)|n|}\right) ;
$$

here $\eta$ is a positive constant, depending on $g_{1}$. From (7), applied for $\epsilon=\eta / 2$, it follows that

$$
g(x)=\sum_{n \neq 0} c_{n}\left(e^{2 \pi i n h}-1\right)^{-1} e^{2 \pi i n x}
$$

defines a function $g \in \Omega_{4}$, such that $\Delta_{h} g-g_{1}=-c_{0}$. This shows that each number $h$ in $\Gamma$ is characteristic w.r.t. $\Omega_{4}$.

Vice versa, let the number $h$ be such, that to each $g_{1} \in \Omega_{4}$ of the form 


$$
g_{1}(x)=\sum_{-\infty}^{\infty} e^{-(2 \pi \delta+\eta)|n|} e^{2 \pi i n x},
$$

there corresponds a $g \in \Omega_{4}$ with $\Delta_{h} g-g_{1}=$ constant. Let

$$
g(x)=\sum_{-\infty}^{\infty} d_{n} e^{2 \pi i n x} \text { with }\left|d_{n}\right| \leqq K_{\eta} e^{-2 \pi \delta|n|},
$$

where $K_{\eta}$ is a positive constant. Then, for $n \neq 0$,

$$
e^{-(2 \pi \delta+\eta)|n|}=\left|d_{n}\left(e^{2 \pi i n h}-1\right)\right| \leqq K_{\eta} e^{-2 \pi \delta|n| \cdot 2|\sin \pi n h|,}
$$

showing that, for each pair of integers $m, n$ with $n \neq 0$,

$$
|n h-m| \geqq \frac{1}{\pi}|\sin \pi n h| \geqq \frac{1}{2 \pi K_{\eta}} e^{-\eta|n|} .
$$

Here, $\eta$ is an arbitrary positive constant, hence, $h \in \Gamma$.

8. A theorem of Skitovic. In this section, we shall give a new proof of the following result due to Skitovic [19].

THEOREM 8.1. Let $a_{v j}(\nu=1,2 ; j=1, \cdots, n)$ be real constants and let $X_{1}, \ldots, X_{n}$ be independent random variables, such that $l_{1}=\sum a_{1 j} X_{j}$ and $l_{2}=\sum a_{2 j} X_{j}$ are independent. Then each $X_{j}$, which occurs in both $l_{1}$ and $l_{2}$, (i.e. $\left.a_{1 j} a_{2 j} \neq 0\right)$, is normally distributed.

We shall need the following lemma, (also used by Skitovic).

Lemma 8.1. Let $X$ be a random variable with characteristic function $\phi(t)$. Let $\epsilon>0$, and suppose that, for $|t|<\epsilon, \phi(t)=e^{P(t)}$, where $P(t)$ is a polynomial. Then $X$ is normally distributed.

This lemma was first stated by Linnik $[11,10]$. In fact, it is an easy consequence of the following result due to Marcinkiewicz $[13,87]$.

Let $X$ be a random variable with cumulative $d . f . F(x)$ and characteristic function $\phi(t)$. Let $0<\epsilon, 0<R \leqq \infty$, and suppose that the restriction of $\phi(t)$ to the interval $-\epsilon<t<+\epsilon$ can be extended to an analytic function $\phi(z)=\phi\left(t+i t^{\prime}\right)$ in the rectangle $|t|<\epsilon,\left|t^{\prime}\right|<R$. Then the integral

$$
\phi(z)=\int_{-\infty}^{\infty} e^{i s x} d F(x)
$$

converges for all complex $z=t+i t^{\prime}$ with $\left|t^{\prime}\right|<R$.

Consequently, (1) defines an analytic extension of the unrestricted func. tion $\phi(t)$ to the strip $\left|t^{\prime}\right|<R$, such that

$$
\left|\phi\left(t+i t^{\prime}\right)\right| \leqq \phi\left(i t^{\prime}\right) .
$$

Under the conditions of Lemma 8.1, this extension is an entire function of the form $\phi(z)=e^{P(z)}$, where $P(z)$ is a polynomial. But then, as was shown by 
Marcinkiewicz $[12,616],(2)$ readily implies that $P(z)$ is at most of second degree, hence, $X$ is normally distributed.

Proof of Theorem 8.1. Permuting the indices, we may assume that

$$
a_{1 j} \neq 0, \quad a_{2 j} \neq 0, \quad(j=1, \cdots, k),
$$

$a_{2 j}=0,(j=k+1, \cdots, q)$ and $a_{1 j}=0,(j=q+1, \cdots, n)$, where $0 \leqq k \leqq q \leqq n$. Let

$$
L_{\nu}=\sum_{j=1}^{k} a_{v j} X_{j},(\nu=1,2) ; \quad Y_{1}=\sum_{j=k+1}^{q} a_{1 j} X_{j} ; \quad Y_{2}=\sum_{q+1}^{n} a_{2 j} X_{j} .
$$

Then $X_{1}, \cdots, X_{k}, Y_{1}, Y_{2}$ are independent, while $l_{\nu}=L_{\nu}+Y_{\nu},(\nu=1,2)$. Let $\phi_{j}(t), \psi_{\nu}(t), f_{\nu}(t)$ denote the characteristic function of $X_{j}, L_{\nu}$ and $Y_{\nu}$, respectively, $(j=1, \cdots, k ; \nu=1,2)$. The joint characteristic function of $l_{1}$ and $l_{2}$ is given by

$$
E\left(e^{i\left(t_{1} l_{1}+t_{2} l_{2}\right)}\right)=\prod_{j=1}^{k} \phi_{j}\left(a_{1 j} t_{1}+a_{2 j} t_{2}\right) f_{1}\left(t_{1}\right) f_{2}\left(t_{2}\right)
$$

and also by

$$
E\left(e^{i t_{1} l_{1}}\right) E\left(e^{\left.i t_{2} l_{2}\right)}=\psi_{1}\left(t_{1}\right) f_{1}\left(t_{1}\right) \psi_{2}\left(t_{2}\right) f_{2}\left(t_{2}\right),\right.
$$

from the independence of $l_{1}$ and $l_{2}$. Any characteristic function $g(t)$ is continuous at $t=0$; moreover, $g(0)=1$. Hence, $f_{\nu}\left(t_{\nu}\right) \neq 0$ for $\left|t_{\nu}\right|<\epsilon_{\nu}$, where $\epsilon_{\nu}$ is a sufficiently small positive number, $(\nu=1,2)$. It follows that, for $\left|t_{1}\right|<\epsilon_{1}$, $\left|t_{2}\right|<\epsilon_{2}$,

$$
\psi_{1}\left(t_{1}\right) \psi_{2}\left(t_{2}\right)=\prod_{j=1}^{b} \phi_{j}\left(a_{1 j} t_{1}+a_{2 j} t_{2}\right)
$$

Replacing (if necessary) $\epsilon_{1}$ and $\epsilon_{2}$ by smaller positive numbers, we have, for $\left|t_{1}\right|<\epsilon_{1},\left|t_{2}\right|<\epsilon_{2}$, that

$$
\left|\arg \psi_{\nu}\left(t_{v}\right)\right|<\pi / 2 ; \quad\left|\arg \phi_{j}\left(a_{1} t_{1}+a_{2 j} t_{2}\right)\right|<\pi / k ;
$$

$(\nu=1,2 ; j=1, \cdots, k)$. Now, the transformation $\zeta=\log z=\log |z|+i$. $\arg z$, with $-\pi<\arg z \leqq+\pi$, is single valued and continuous in the sector $|\arg z|<\pi$ and such that $\log \left(z_{1} z_{2} \cdots z_{p}\right)=\log z_{1}+\cdots+\log z_{p}$ whenever $\left|\arg z_{j}\right|<\pi / p$ $(j=1, \cdots, p)$. Hence, for $\left|t_{1}\right|<\epsilon_{1},\left|t_{2}\right|<\epsilon_{2}$,

$$
\log \psi_{1}\left(t_{1}\right)+\log \psi_{2}\left(t_{2}\right)=\sum_{j=1}^{k} \log \phi_{j}\left(a_{1 j} t_{1}+a_{2 j} t_{2}\right),
$$

where $\log \psi_{1}(t)$ is continuous at $t=0$. Putting

$$
g_{0}(t)=-\log \psi_{1}(t), \quad g_{j}(t)=\log \phi_{j}\left(a_{1 j} t\right), \quad(j=1, \cdots, k),
$$

we have, for $\left|t_{1}\right|<\epsilon_{1},\left|t_{2}\right|<\epsilon_{2}$, that 


$$
\sum_{j=0}^{k} g_{j}\left(t_{1}+b_{j} t_{2}\right)=\log \psi_{2}\left(t_{2}\right),
$$

where $b_{0}=0, b_{j}=a_{2 j} / a_{1 j} \neq 0,(j=1, \cdots, k)$. Moreover, $g_{0}(t)$ is bounded in a neighborhood of $t=0$. The right hand side of (3) being independent of $t_{1}$, it follows from assertion (iii) of Theorem 6.1, applied for $A=\left\{|t|<\epsilon_{1}\right\}$, $C=\left\{|t|<\epsilon_{2}\right\}$, (cf. also the following lemma), that the function $g_{0}(t)$ $=-\log \psi_{1}(t)$ is equal to a polynomial throughout the interval $|t|<\epsilon_{1}$. Hence, from Lemma 8.1, the random variable $L_{1}$ is normally distributed. But the normal law is closed under decomposition, Cramér $[5,52]$, thus, the normality of $L_{1}=\sum a_{1 j} X_{j}$ implies the normality of $a_{1 j} X_{j}$ and, hence, of $X_{j},(j=1, \cdots, k)$.

REMARK. It is easily seen, Skitovic $[19$, p. 200], that Lemma 8.1 continues to hold when $X$ and $t$ are replaced by vectors in $E_{m}$. Further, in $E_{m}$, the normal law is closed under decomposition, Cramér $[5,112]$. Consequently, the above proof also yields the generalization of Theorem 8.1 to independent random vectors $X_{1}, \cdots, X_{n}$ in $E_{m}$. On the other hand, as was shown by Skitovic [19], it is not difficult to obtain this generalization directly from Theorem 8.1.

For the benefit of those readers who are mainly interested in the proof of Theorem 8.1 we shall give a separate proof of the following corollary of Theorem 6.1, which will suffice in proving Theorem 8.1.

Lemma. Let $\alpha<\beta, \epsilon>0$ be real constants. Suppose that, for $t_{1} \in I=\{\alpha<t<\beta\}$ and $\left|t_{2}\right|<\epsilon$,

$$
\sum_{j=0}^{k} g_{j}\left(t_{1}+b_{j} t_{2}\right)=\left(\text { polynomial in } t_{1}\right)=\sum_{r=0}^{N\left(t_{2}\right)} a_{r}\left(t_{2}\right) t_{1} .
$$

Here, the $b_{j}$ are real constants, $b_{j} \neq b_{0}=0,(j=1, \cdots, k)$. Further, $g_{j}(t)$ is a complex-valued function defined for $\alpha-\left|b_{j}\right| \epsilon<t<\beta+\left|b_{j}\right| \epsilon,(j=0, \cdots, k)$. Then, in $I$, the function $g_{0}(t)$ is equal to a polynomial if (and only if) it is bounded on some nondegenerate subinterval $I_{1}$ of $I$.

Proof. The assertion is obvious for $k=0$. Let $k \geqq 1$, and suppose that the lemma holds true when $k$ is replaced by $k-1$. Let $\left|I_{1}\right|$ denote the length of $I_{1}, 0<\left|I_{1}\right| \leqq \beta-\alpha$, let $\delta=\operatorname{Min}\left(\left|I_{1}\right|, 1 / 2\left|b_{k}\right| \epsilon\right)$, and let $h$ be any fixed positive number $<\delta$. From $0<h<\delta \leqq 1 / 2\left|b_{k}\right| \epsilon$, we have

$$
-\left|b_{j}\right| \epsilon / 2<\left(1-b_{j} / b_{k}\right) h<h+\left|b_{j}\right| \epsilon / 2 \text {. }
$$

Hence, the function

$$
g_{j}^{*}(t)=g_{j}\left(t+\left(1-b_{j} / b_{k}\right) h\right)-g_{j}(t), \quad(j=0, \cdots, k)
$$

is defined for $\alpha-\left|b_{j}\right| \epsilon / 2<t<\beta-h+\left|b_{j}\right| \epsilon / 2$, especially, $g_{0}{ }^{*}(t)=g_{0}(t+h)-g_{0}(t)$ is defined throughout $I^{*}=\{\alpha<t<\beta-h\}$. Further, from $0<h<\delta \leqq\left|I_{1}\right|$, the function $g_{0}{ }^{*}(t)$ is bounded on some nondegenerate subinterval $I_{1}^{*}$ of $I^{*}$. 
If $\left|t_{2}\right|<\epsilon / 2$ then $\left|t_{2}-h / b_{k}\right|<\epsilon / 2+\delta /\left|b_{k}\right| \leqq \epsilon$. Thus, if $\phi\left(t_{1}, t_{2}\right)$ denotes the left hand side of (4), we have, for $\left|t_{2}\right|<\epsilon / 2, t_{1} \in I^{*}$, that

$$
\left.\psi\left(t_{1}, t_{2}\right)=\phi\left(t_{1}+h, t_{2}-h / b_{k}\right)-\phi\left(t_{1}, t_{2}\right)=\text { (polynomial in } t_{1}\right) .
$$

Moreover, from (4) and (5), (especially, $g_{k}^{*}(t)=0$ ),

$$
\psi\left(t_{1}, t_{2}\right)=\sum_{j=0}^{k-1} g_{j}^{*}\left(t_{1}+b_{j} t_{2}\right)
$$

Hence, by induction, (replacing $g_{j}(t)(j=0, \cdots, k), I, I_{1}$ and $\epsilon$ by $g_{j}^{*}$ $(j=0, \cdots, k-1), I^{*}, I_{1}{ }^{*}$ and $\epsilon / 2$, respectively), it follows that, in $I^{*}$ $=\{\alpha<t<\beta-h\}$, the function $g_{0}{ }^{*}(t)=\Delta_{h} g_{0}(t)$ is equal to a polynomial. In fact, this is true for each positive number $h<\delta$.

Let $h^{\prime}$ be fixed, $0<h^{\prime}<\delta / 2$. Then $\Delta_{h^{\prime}} g_{0}(t)\left(\alpha<t<\beta-h^{\prime}\right)$ is equal to a polynomial, hence, there exists a polynomial $P(t)$, such that $\Delta_{h^{\prime}} g_{0}(t)=\Delta_{h^{\prime}} P(t)$, $\alpha<t<\beta-h^{\prime}$. Let

$$
f_{0}(t)=g_{0}(t)-P(t), \quad(\alpha<t<\beta),
$$

thus, $\Delta_{h} f_{0}(t)=0, \alpha<t<\beta-h^{\prime}$. Moreover, for $0<h<\delta / 2$, the function $C(h, t)$ $=\Delta_{h} f_{0}(t)=\Delta_{h} g_{0}(t)-\Delta_{h} P(t),(\alpha<t<\beta-h)$ is equal to a polynomial, such that

$$
\Delta_{h^{\prime}} C(h, t)=\Delta_{h^{\prime}} \Delta_{h} f_{0}(t)=\Delta_{h} \Delta_{h^{\prime}} f_{0}(t)=0,
$$

$\left(\alpha<t<\beta-h-h^{\prime}\right.$ with $\left.h+h^{\prime}<\delta \leqq \beta-\alpha\right)$. It follows that, for $0<h<\delta / 2$, the polynomial $C(h, t)=\Delta_{h} f_{0}(t)$ is equal to a constant $C(h)$. From

$$
\Delta_{h+h^{\prime}} f_{0}(t)=\Delta_{h} f_{0}(t)+\Delta_{h^{\prime}} f_{0}(t+h),
$$

$\left(h \geqq 0, h^{\prime} \geqq 0, \alpha<t<\beta-h-h^{\prime}\right)$, we easily see that $\Delta_{h} f_{0}(t)(\alpha<t<\beta-h)$ is equal to a constant $C(h)$ for any number $h$ with $0 \leqq h<\beta-\alpha$, in such a way that

$$
C\left(h+h^{\prime}\right)=C(h)+C\left(h^{\prime}\right),
$$

for $h \geqq 0, h^{\prime} \geqq 0, h+h^{\prime}<\beta-\alpha$. Because $f_{0}(t)=g_{0}(t)-P(t)$ is bounded on a nondegenerate interval $I_{1} \subset I$, we have that $C(h)=\Delta_{h} f_{0}(t)$ is bounded for $0 \leqq h \leqq \eta$, when $\eta$ is a sufficiently small positive number. This, together with (6), easily implies the existence of a constant $\gamma$, such that $C(h)=\gamma h$ for $0 \leqq h<\beta-\alpha$. But $C\left(h^{\prime}\right)=\Delta_{h^{\prime}} f_{0}(t)=0$, hence, $\gamma=0$ and

$$
f_{0}(t+h)-f_{0}(t)=\Delta_{h} f_{0}(t)=C(h)=\gamma h=0,
$$

for $0<h<\beta-\alpha, \alpha<t<\beta-h$. It follows that $f_{0}(t)$ is constant in $\alpha<t<\beta$, thus, $g_{0}(t)=P(t)+f_{0}(t)$ is equal to a polynomial.

\section{REFERENCES}

1. R. P. Boas Jr., Functions which are odd about several points, Nieuw Archief voor Wiskunde (3) vol. 1 (1953) pp. 27-32. 
2. N. G. de Bruijn, Functions whose differences belong to a given class, Nieuw Archief voor IViskunde (2) vol. 23 (1951) pp. 194-218.

3. - A difference property for Riemann integrable functions and for some similar ciasses of functions, Proc. Kon. Ned. Akad. Wetenschappen Amsterdam (A) vol. 55 (1952) pp. $145-151$.

4. T. Carleman, Les fonctions quasi-analytiques, Paris, 1926.

5. H. Cramér, Random variables and probability distributions, Cambridge, 1937.

6. P. Erdös and M. Golomb, Functions which are symmetric about several points, Nieuw Archief voor Wiskunde (3) vol. 3 (1955) pp. 13-19.

7. H. Hahn, Über Funktionen mehrerer Veränderlicher die nach jeder einzelnen Veränderlichen stetig sind, Math. Zeit. vol. 4 (1919) pp. 306-313.

8. H. Kestelman, On the functional equation $f(x+y)=f(x)+f(y)$, Fund. Math. vol. 34 (1947) pp. 144-147.

9. J. F. Koksma, Diophantische Approximationen, Berlin, 1936.

10. H. Lebesgue, Sur les fonctions représentables analytiquement, J. Math. Pures Appl. vol. 1 (1905) p. 201.

11. Y. V. Linnik, On some equally distributed statistics, (Russian), Doklady Akademii Nauk SSSR. vol. 89 (1953) pp. 9-11.

12. J. Marcinkiewicz, Sur une propriété de la loi de Gauss, Math. Zeit. vol. 44 (1938) pp. 612-618.

13. - Sur les fonctions independantes III, Fund. Math. vol. 31 (1938) pp. 86-102.

14. N. E. Nörlund, Differenzenrechnung, Berlin, 1924.

15. A. Ostrowski, Über die Funktionalgleichung der Exponentialfunktion und verwante Funktionalgleichungen, Jber. Deutschen Math. Verein. vol. 38 (1929) pp. 54-62.

16. S. Ruziewicz, Contribution a l'etude des ensembles de distances de points, Fund. Math. vol. 7 (1925) pp. 141-143.

17. W. Sierpinsky, Démonstration elémentaire du théorème sur la densité des ensembles, Fund. Math. vol. 4 (1923) pp. 161-171.

18. - Hypothèse du continu, Warsaw, 1934, p. 135.

19. V. P. Skitovic, Linear forms of independent random variables and the normal distribution law, (Russian), Izvestiya Akademii Nauk SSSR. Seriya Matematičeskaya vol. 18 (1954) pp. 185-200.

20. H. Steinhaus, Sur les distances des points dans les ensembles de mesure positive, Fund. Math. vol. 1 (1920) pp. 93-104.

Purdue University,

LAFAYETTE, IND. 